\title{
Density Functional Theory Calculations on Interface Structures and Ad- sorption Properties of Graphenes: A Review
}

\author{
Z. M. Ao and Q. Jiang*
}

Key Laboratory of Automobile Materials, Ministry of Education, and School of Materials Science and Engineering, Jilin

University, Changchun 130022, China

\begin{abstract}
This review covers interface properties and adsorption behaviors of graphene underlying density functional theory (DFT) simulations and their relevance in evaluation, developing and discovering new materials for gas sensors and hydrogen storage materials. It is intended to be of interest for both experimentalists and theorists to expand application fields of graphene.
\end{abstract}

\section{INTRODUCTION}

Graphene is a single layer of graphite in a hexagonal structure, or an individual sheet of $\mathrm{sp}^{2}$-hybridized carbon bound in two dimensions. For more than six decades, scientists have presumed that a single-layer graphene sheet (one atom thick) could not exist in its free state based on the reasoning that its planar structure would be thermodynamically unstable [1]. Somewhat surprisingly, graphene was experimentally fabricated for the first time in 2004, which was prepared by mechanical exfoliation of small mesas from highly oriented pyrolytic graphite, and graphene was found to have promising electronic properties [2]. This discovery has triggered enormous amount of studies on graphene since then [3-6]. Several groups worldwide have recently succeeded in obtaining isolated graphene sheets with various preparing techniques [7-11].

Several unique electronic properties associated with these $2 D$ crystals have been discovered [12]. Research into the electronic properties of graphene has followed two parallel courses. One course involves the study of mechanically exfoliated graphene sheets [2,3,13-15]. In this research, graphene flakes (typically in micron size) are mechanically peeled from a bulk graphite crystal onto a supporting substrate. Once a single graphene sheet is subsequently located by optical microscopy, metal contacts are attached for transport studies [3,4,14-17]. In the second research avenue, graphene is directly grown on large area insulating or semiconducting substrates. Once grown, the films are lithographically patterned and metal contacts are applied to make electronic devices $[9,18,19]$. Graphenes produced in this way are referred to as epitaxial graphenes (EG). Mechanically exfoliated graphene flakes have been used to study a variety of fundamental graphene properties. These flakes have been shown to exhibit $2 D$ transport property characteristics of the

\footnotetext{
*Address correspondence to this author at the Key Laboratory of Automobile Materials, Ministry of Education, and School of Materials Science and Engineering, Jilin University, Changchun 130022, China; Tel: 86-431-85095371; Fax: 86-431-85095876; E-mail: jiangq@jlu.edu.cn
}

chiral massless Dirac electrons [20] expected for an isolated graphene sheet. These include an unusual half-integer quantum Hall effect and a non-zero Berry's phase [3,14].

Recently, Li et al. developed a facile approach to largescale production of aqueous graphene dispersions, which makes it possible to process graphenes using low-cost solution processing techniques $[21,22]$. Therefore, it would be of interest of opening up opportunities for its further applications.

Due to the instability of a freestanding graphene (it has an intrinsic $3 D$ structure or ripples [23]), graphenes used as devices are generally located on a substrate [13], such as on the common gate dielectric $\alpha-\mathrm{SiO}_{2}$ substrate [24]. Substrate-induced structural distortion [25] and bandgap opening [9,26], adsorbates [27-29], local charge disorder [30], atomic structure at edges [31,32], and even atomic scale defects [33], could be very important for transport properties for graphene/substrate systems. To find these properties, a graphene on a $\mathrm{SiO}_{2}$ [2] or $\mathrm{SiC}$ [9] substrate has been experimentally studied, while graphenes on substrates of $\mathrm{Cu}(111)$ [26], $h$-BN [26], Ni(111) [34], Pt(111) [35], and $\operatorname{Ir}(111)$ [36] have been simulated using the first-principles density functional theory (DFT).

It is known that carbon nanotubes have good sensor properties [4]. Recently, graphenes as highly sensitive gas sensors were also reported [5,37]. It was shown that the increase in graphene charge carrier concentration induced by adsorbed gas molecules could be utilized to make highly sensitive sensors, even with the possibility of detecting individual molecules. The sensor property is based on changes in the resistivity due to molecules adsorbed on graphene sheet that acts as donors or acceptors. The sensitivity of $\mathrm{NH}_{3}, \mathrm{CO}$, and $\mathrm{H}_{2} \mathrm{O}$ up to $1 \mathrm{ppb}$ (parts per $10^{9}$ ) was demonstrated, and even the ultimate sensitivity of an individual molecule was suggested for $\mathrm{NO}_{2}$. These excellent sensor properties of graphenes are due to the following reasons: (1) Graphene is a single atomic layer of graphite with surfaces only, this can maximize the interaction between the surface dopants and adsorbates; (2) Graphene has much smaller band gap energy, $E_{\mathrm{g}}$, than carbon nanotube $(\mathrm{CNT})$. Hence, it has extremely 
low Johnson noise $[3,4,14]$. As results, a little change of carrier concentration can cause a notable variation of electrical conductivity; and (3) Graphene has limited crystalline defects $[3,4,14,38]$, which ensures a low level of excess noise caused by their thermal switching [39].

Furthermore, preliminary works [29] indicated that graphenes may have promising physisorption properties for hydrogen due to the following characteristics: (i) a large surface for hydrogen adsorption, (ii) economical and scalable production [21] and (iii) the strongest material ever measured [40].

With state-of-art computer simulations, it is believed that condensed materials can be understood at the atomic level. In the simulation, the simulator builds a model of a real system and explores its properties. The model is a mathematical one and the exploration is done on a computer. In many ways, simulation studies share the same mentality as experimental ones. However, simulations allow absolute control over the experimental parameters and access to the outcomes in details. These strengths have been exploited for the last fifty years since the introduction of computation algorithms that allows one to calculate the properties of materials based on the first-principles in light of fundamental physics outlined in Schrödinger equation without free parameters.

Therefore, in this review, we will briefly outline the fundamentals underpinning DFT in section 2, a commonly used technique for modeling molecular and solid systems as it is faster than wave function based ab initio methods, albeit at the cost of reduced accuracy. We will then discuss how this technique may be applied to calculate graphene/substrate interface and gas molecules adsorbed on graphene in sections 3 and 4 . At last, we give a simplified summary to denote main results of this review.

\section{THEORETICAL BACKGROUND: DENSITY FUN- TIONAL THEORY}

The properties of a material are determined by behaviors of electrons that comprise bond strengths of the system in question. In DFT, the ground state of an interaction electron gas is mapped onto the ground state of a non-interacting electron gas, which experiences an effective potential. This potential is in contrast to other techniques such as Hartree-Fock, which solves a full set of wave functions in the Schrödinger equation. This mapping in principle gives exact ground-state properties (such as the cohesive energy, lattice parameters and phonon spectra). The Hamiltonian that describes the electron gas density is,

$$
\hat{H}=\hat{T}+\hat{V}_{e e}+\sum_{i=1}^{N} V_{e x t}\left(r_{i}\right)
$$

where $\hat{T}$ and $\hat{V}_{e e}$ are the kinetic and electron-electron interaction operators, respectively, and $V_{\text {ext }}$ is the external potential. The two basic theorems of DFT are: (i) There exists a functional $F[n]$ of the electronic density $n$ that represents the ground state energy $E_{\mathrm{GS}}$, (ii) The only density, which gives an energy being equal to that of the ground state, is the electronic density of the ground state $n_{\mathrm{GS}}$, with all other densities giving higher energies, $\int d r V_{e x t}(r) n_{G S}(r)+F\left[n_{G S}\right] \equiv E_{G S}$. The functional $E[n]$ thus satisfies a variational principle.

A separation of the Hamiltonian firstly devised by Kohn and Sham is used [41], wherein we can write the energy as a sum of functionals that depends on electron density,

$$
E[n(r)]=T_{0}[n(r)]+\int d r n(r)\left[V_{e x t}(r)+1 / 2 \Phi(r)\right]+E_{X C}[n(r)]
$$

where $T_{0}$ is the kinetic energy of a system with $n$ in the absence of electron-electron interactions, $\Phi$ is the classical Coulomb potential and $E_{\mathrm{XC}}$ is the exchange and correlation energy. The exchange energy is the contribution to the energy that results from the Pauli principle that no two electrons of the same spin energy can reside in one state. The correlation energy is the contribution to the energy from the Coulomb repulsion that an electron experiences from all other electrons.

$E_{\mathrm{XC}}$ thus depends on the electronic density. As it is not known how to solve the n-body problem exactly, we have no ideal a priori how $E_{\mathrm{XC}}[n]$ behaves. The oldest and simplest way of solving the variational equations is the local-density approximation (LDA) [42]. This assumes that the exchange-correlation potential for an electron is equal to the exchange-correlation potential of an electron in a uniform gas of interaction electrons, and can be inserted into the above equation as,

$$
E_{X C}^{L D A}=\int d r n(r) \varepsilon_{X C}[n(r)]
$$

where $\varepsilon_{\mathrm{XC}}[n(r)]$ is the exchange-correlation energy density of a homogeneous electron gas. In actuality, although LDA is surprisingly accurate for many systems, it has been surpassed by newer and more sophisticated functionals. A useful scheme for classifying all of these functionals is to divide them into classes based on the types of variables that are involved, the so-called "Jacob's ladder" of functionals [43]. At the lowest level, the exchange-correlation functional depends solely on the electron density $n, e . g$. the LDA. The next level up is the generalized gradient approximations (GGA) [44-48].

Historically the GGAs originated in the presumption that as real systems are not homogeneous, then improvements to the LDA can be made by treating the electron gas as slowly varying and expanding to second order, the gradient expansion approximation (GEA) [49],

$$
U^{G E A}[n(r)]=\int\left\{a[n(r)]+b[n(r)]|\nabla n(r)|^{2}+\cdots\right\}
$$

Unfortunately this expansion does not lead to improvements. As the real electron gas varies rapidly, the expansion breaks down at large distance due to large oscillations in the electron density [50]. The GEA is an improvement at shorter 
distances however. In order to utilize the advantages of the GEA, there needs to a truncation at the point where unphysical oscillations in the exchange-correlation density manifest. This truncation is known as the GGA. Placing exchange and correlation into separate terms, for any GGA the exchange energy may be written as,

$$
E_{X}^{G G A}=\int d^{3} r n e_{x}^{u n i f}(n) F_{X}[s(r)]
$$

where $F_{\mathrm{X}}[s(r)]$ is the factor of exchange enhancement caused by the GGA and $s(r)=|\nabla n(r)| / 2 k_{F} n$ is a dimensionless density gradient parameter being dependent on the Fermi wavenumber of the material $k_{\mathrm{F}}$ [48]. To recover the correct uniform gas limit, set $F_{\mathrm{X}}(0)=1$. For the correlation energy there is simply a renormalization of all radii such that $r$ is now set to $r_{\mathrm{s}}$. The full GGA correction to the exchange-correlation energy $E_{\mathrm{XC}}$ is,

$$
E_{X C}^{G G A}[n]=\int d^{3} r n e_{x}^{u n i f}(n) F_{X C}\left(r_{s}, s\right)
$$

and is dependent on both $n$ and $\nabla n$. Different flavors of GGA are simply choices on how this truncation is performed. The Perdew-Wang (PW91) GGA is an analytic fit to this nonempirical GGA $[46,49]$. However, the parameterisation is complex. For example, the exchange enhancement factor in this functional is,

The Perdew, Burke and Enzerhof (PBE) GGA [48] is a modification of this nonempirical functional that only satis- of the exchange correlation hole and thus are implicit functional of the density. Several meta-GGAs have been developed, but the only nonempirical functional is the TPSS functional of Tao, Perdew, Staroverov, and Scuseria [51].

Higher rungs up the ladder of functionals would introduce more nonlocalities. For example, it has been posited that the next rung up would be the so-called "hyper-GGA" functionals which would include exact exchange energy density [43]. Currently there are not any nonempirical formulations of a hyper-GGA. "Hybrid" functional, which admixes a portion of exact Hartree-Fock exchange into the exchange-correlation functional [52,53], can be regarded as analogous functionals to the hyper-GGA. These tend to be the functionals of choice for the chemistry community and often are the most accurate functionals for calculations of barrier heights. However these functionals are optimized semi-empirically on a specific set of small molecules, the transferability to other systems may thus be constrained.

\section{THE ATOMIC STRUCTURE OF GRAPHENE/ $\alpha-S_{2} O_{2}$ INTERFACE}

Moore's law, a scaling rule of thumb turned into self-fulfilling prophecy, has dictated the exponential growth of the semiconductor industry over the last four decades [54]. To keep the law being valid in the future development, carbon based electronics offers one of the most promising options to replace silicon. In the past, great attention has been paid to CNTs due to their intriguing electronic properties $[55,56]$. Their random orientation and spatial distribution however inhibit their introduction into the reality application where more than one billion devises need to be connected.

$$
F_{X}[s(r)]=\frac{1+0.19645 s \sinh ^{-1}(7.7956 s)+\left(0.2743-0.1508 e^{-100 s^{2}}\right) s^{2}}{1+0.19654 s \sinh ^{-1}(7.7956 s)+0.004 s^{4}}
$$

fies conditions, which are energetically relevant. For instance, the exchange enhancement factor in the PBE is,

$$
F_{X}(s)=1.804-\frac{0.804}{1+0.24302 s^{2}},
$$

which leads to a much smaller and more transparent parameterisation.

The next rung in the ladder of increasing accuracy of density functionals is the meta-GGAs. As the LDA is a functional that depends only on $n$, and the GGAs are functionals that depend on $n$ and $\nabla n$, meta-GGAs are functionals that are often the form [51],

$$
E_{X C}^{M G G A}[n]=\int d^{3} r \varepsilon_{X C}^{M G G A}\left(n, \nabla n, \nabla^{2} n, t\right)
$$

where $t$ is the Kohn-Sham orbital kinetic energy densities $t_{\sigma}(r)=\frac{1}{2} \sum_{i}^{o c c}\left|\nabla \phi_{i \sigma}\right|^{2}$, which appears in the Taylor expansion
An alternative solution could be graphene, which has excellent electronic properties with carrier mobilities between 3.000 and $27.000 \mathrm{~cm}^{2} \mathrm{Vs}^{-1}$ at room temperature [2,7] and can be taken as an extremely promising material for nanoelectronic devices.

Experimentally, controlling the environment of graphene in a device configuration is difficult. Graphene on the common gate dielectric, $\alpha-\mathrm{SiO}_{2}$, is subject to the effects of trapped oxide charges [24], which is highly dependent on sample preparation. In addition, graphene devices are typically fabricated using electron beam lithography, which may modify electronic transport properties [30]. Meanwhile, experimental data on electronic properties of graphene have been obtained mainly from a mono- or a few layer graphene on $\mathrm{SiO}_{2}$ wafers [2,7] or decomposed intrinsic silicon carbide [9]. The properties of graphene-based devices are likely to be controlled by the application of the external gate voltage [15]. It is well known that the electric field strength $F$ can effectively modify both electronic and transport properties of low dimensional systems, e.g., 1D quantum wires [57] and CNTs $[58,59]$. These influences have been studied by tight-binding 

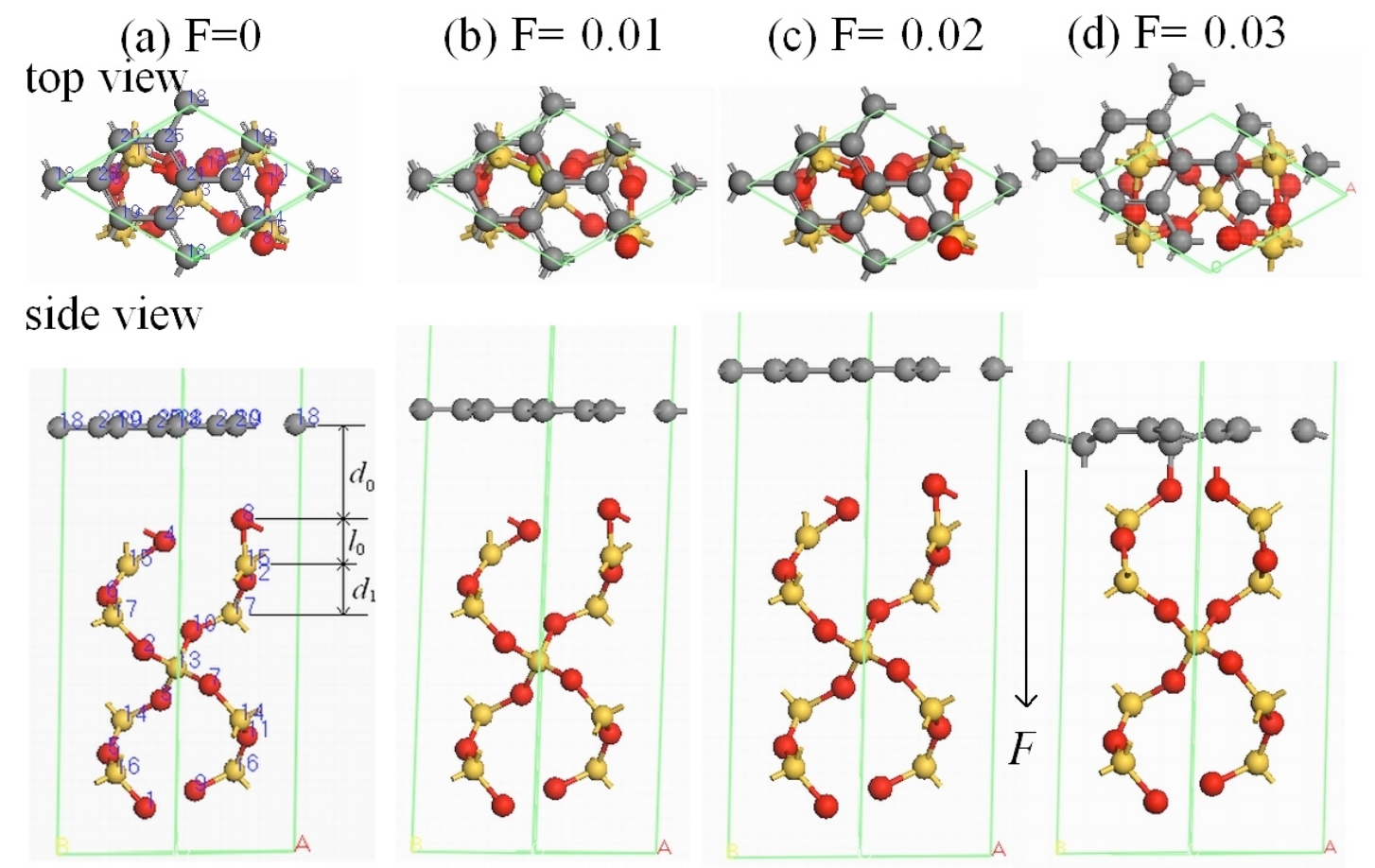

Fig. (1). Atomic stacking sequence of graphene/SiO $\mathrm{S}_{2}$ system under $F=0$ (a), $F=0.01$ au (b), $F=0.02$ au (c), and $F=0.03$ au (d). The yellow, gray, and red spheres show $\mathrm{Si}, \mathrm{C}$, and $\mathrm{O}$ atoms, respectively. The numbers are serial numbers of atoms in the simulation. The direction of the added $F$ is pointed out by the arrowhead. (Reproduced with permission from Ref. [6]. Copyright 2008, IOP).

model and experiments $[60,61]$. It is found that the band structure and the density of state (DOS) are very sensitive to the changes of the direction, and the period of a modulated $F$. The corresponding variation of atomic structure of graphene is essential for the electrons carrier mobility and may cause structure breakage when $F$ value is large [61].

In this section, DFT calculations, which could avoid the effects of experimental environment, experimental technology, and so on, are described to determine atomic structure changes of graphene/ $\alpha-\mathrm{SiO}_{2}(0001)$ system under different $F$ in downward direction normal to the interface. Further, the determination of its charge distribution and electronic density difference on the interface are shown and their effects on the atomic structure changes are discussed.

DFT calculations are performed with $\mathrm{DMol}^{3}$ code [62]. GGA with the revised Perdew-Burke-Emzerhof (RPBE) method is used as the exchange correlation function [63]. The DFT semicore pseudopotentials (DSPP) core treat method [62] is implemented for relativistic effects, which replaces core electrons by a single effective potential. To ensure that the results of the calculations are directly comparable, identical conditions are employed for all systems. The $k$-point is set to $6 \times 6 \times 1$ for all simulations, which brings out the convergence tolerance of energy of $2.0 \times 10^{-5}$ hartree $(1$ hartree $=27.2114 \mathrm{eV}$ ), maximum force of 0.004 hartree/ $\AA$, and maximum displacement of $0.005 \AA$.

The initial atomic structure of graphene/ $\alpha-\mathrm{SiO}_{2}(0001)$ is established from bulk $\alpha-\mathrm{SiO}_{2}$ and graphite structures, and a distance between graphene and $\mathrm{SiO}_{2}$ surface $d_{0}=3.000 \AA$ is taken as initial value [9] where simulation cell parameters of graphene and $\mathrm{SiO}_{2}$ substrate are $a=b=4.920 \AA, \alpha=\beta=90$ ${ }^{\circ}, \gamma=120^{\circ}$ and $a=b=4.910 \AA, \alpha=\beta=90^{\circ}, \gamma=120^{\circ}$, respectively. Thus, the lattice mismatch is in the range of $0.3 \%$, and graphene and $\mathrm{SiO}_{2}$ substrate match each other well, which is shown in Fig. (1). A vacuum width of $12 \AA$ is added above graphene layer. A slab with $4 \mathrm{O}-\mathrm{Si}-\mathrm{O}$ monolayers is taken as the bulk surface where atoms of the lowest $\mathrm{SiO}_{2}$ monolayer are kept fixed, which is enough to stand for the bulk surface [64]. The initial atomic structure after relaxation is shown as Fig. (1a).

To probe the effects of $F$, an electric field with different intensities of $F=0.01,0.02$ and 0.03 au $(1 \mathrm{au}=51 \mathrm{~V} / \AA)$ in the downward direction normal to the interface is added to the relaxed system, as shown in Fig. (1). The charge distributions and electronic density differences of graphene/ $\mathrm{SiO}_{2}$ interface are carried out by Mulliken charge analysis and electron density deformation analysis, which are performed using a projection of a linear-Combination of Atomic Orbitals (LCAO) basis and specify quantities such as atomic charge, bond population, charge transfer etc.

The atomic structures of graphene/ $\mathrm{SiO}_{2}$ interface under different $F$ are shown in Fig. (1). The corresponding structure parameters are given in Table 1. It is found that the atomic structure is dependent on $F$. $d_{0}$ increases as $F$ increases from $F=0$ to $F=0.02$ au. However, as $F$ increases further, $d_{0}$ drops greatly from $3.587 \AA$ at $F=0.02$ au to $1.452 \AA$ at $F=0.03$ au where $\mathrm{C}-\mathrm{O}$ bond is formed, as shown in Fig. (1d). This is in agreement with the change of the atomic stacking sequence of the system under $F$ from 0 to 0.03 au where graphene layer moves from upwards to downwards. Meanwhile, the atoms in several surface layers 
Table 1. Results for the Atomic Structures of Graphene/ $\alpha-\mathrm{SiO}_{2}(0001)$ Interface Under Different $F$. $d_{0}$ is the Equilibrium Distance between $\mathrm{SiO}_{2}$ Substrate and Graphene Overlayer, $d_{1}$ is the Distance between the First and the Second Si Atom Layers, $l_{0}$ is the Length of Si-O Bond at Surface or Interface, $l_{\mathrm{C}-\mathrm{C}}$ is Average Bond Length of $\mathrm{C}-\mathrm{C}$

\begin{tabular}{|c|c|c|c|c|}
\hline & $\boldsymbol{F}=\mathbf{0} \mathbf{~ a u}$ & $\boldsymbol{F = 0 . 0 1} \mathbf{a u}$ & $\boldsymbol{F = 0 . 0 2} \mathbf{~ a u}$ & \multicolumn{1}{c|}{$\mathbf{0 . 0 3} \mathbf{~ a u}$} \\
\hline \hline$d_{0}(\AA)$ & 3.227 & 3.273 & 3.587 & 1.452 \\
\hline$d_{1}(\AA)$ & 1.777 & 1.815 & 1.989 & 1.980 \\
\hline$l_{0}(\AA)$ & 1.640 & 1.641 & 1.400 & 1.660 \\
\hline$l_{\mathrm{C}-\mathrm{C}}(\AA)$ & 1.402 & 1.401 & 1.435 \\
\hline
\end{tabular}

(a) $\mathrm{F}=0$

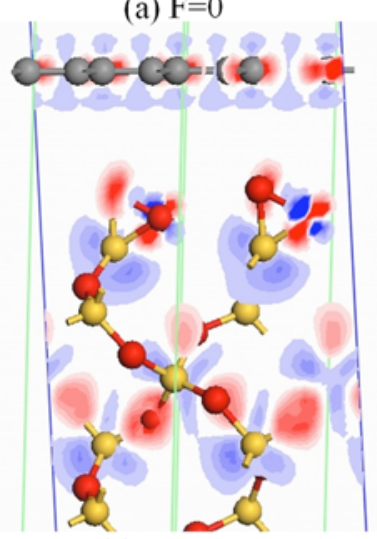

(c) $\mathrm{F}=0.02$

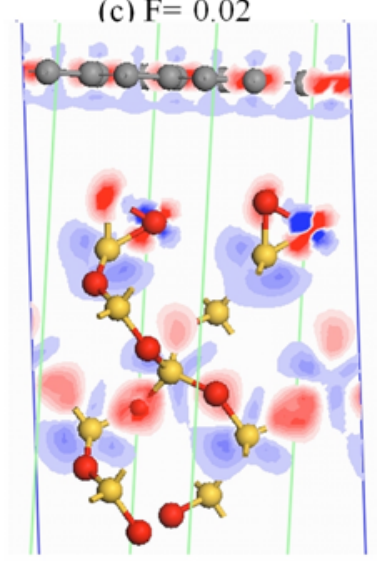

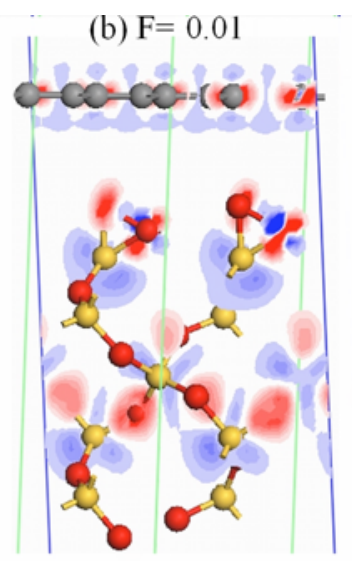

(d) $\mathrm{F}=0.03$

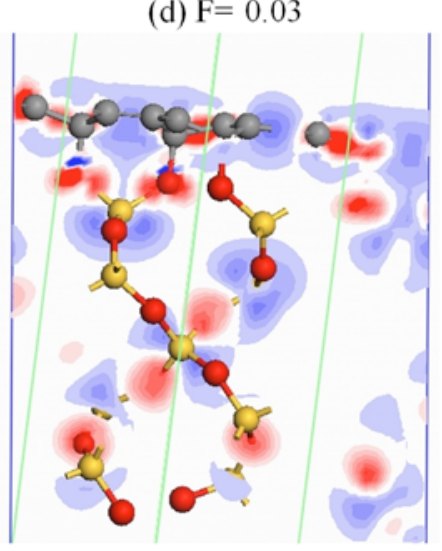

Fig. (2). The images of the electron density difference for graphene/ $\mathrm{SiO}_{2}$ system under different $F$ in unit au. The red and blue regions show the electron accumulation and loss. (Reproduced with permission from Ref. [6]. Copyright 2008, IOP).

of the substrate always move upwards as $F$ increases. This induced by electrons accumulation in the interface region that would cause upwards force in $F$, while graphene layer also moves upwards with interaction of substrate. This change tendency is also observed in the trajectory of simulation where all atoms both in graphene and interface region of $\mathrm{SiO}_{2}$ substrate move upwards for $F \leq 0.02$ au. At $F=0.03 \mathrm{au}$, all the atoms firstly move upwards. However, the motion speed of the substrate atoms is higher than that of $\mathrm{C}$ atoms in graphene. When the $\mathrm{C}-\mathrm{O}$ bond is formed, graphene layer is pulled down where $d_{0}$ decreases at $F=0.03$ au.

Fig. (1d) shows that graphene moves left because of the strong interaction with the substrate $\mathrm{O}$ atoms, which are in

atop sites of graphene. Other structure parameters of $d_{1}, l_{0}$ and $l_{\mathrm{C}-\mathrm{C}}$ are also probed and displayed in Table $\mathbf{1}$, which indicate that $d_{1}$ increases as $F$ varies from 0 to 0.02 au. Although as $F$ reaches 0.03 au, $d_{1}$ decreases, the drop of $d_{1}$ is much smaller than that of $d_{0}$. For $l_{0}$, as $F$ varies from 0 to $0.03 \mathrm{au}$, it increases and has a large upward jump at $F=$ 0.02-0.03 au. In addition, $l_{\mathrm{C}-\mathrm{C}}$ decreases as $F$ increases, but has a tempestuously increase when $F=0.03$ au. Thus, the $\mathrm{C}-\mathrm{O}$ bond formation mainly affects the atomic structure in the interface region, and has only a little effect on other atoms even that in the second layer.

The variation of $l_{\mathrm{C}-\mathrm{C}}$ listed in Table $\mathbf{1}$ is consistent with literature data [65], where a softening of in-plane phonons of the adsorbed graphene compared to that of graphite is present. The softening implies that the $\mathrm{C}-\mathrm{C}$ bond is weakened, which, in turn, strengthens bonds to the substrate. The similar phenomenon is found for the system under $F=0.03 \mathrm{au}$, where $l_{\mathrm{C}-\mathrm{C}}=1.435 \AA$ and $d_{0}=1.452 \AA$ with strong interface interaction, which is longer than $l_{\mathrm{C}-\mathrm{C}}=1.420 \AA$ and shorter than interlayer distance $3.400 \AA$ in graphite [66]. However, the inverse phenomenon is found for the structure under $F=$ $0,0.01$, and 0.02 au where $l_{\mathrm{C}-\mathrm{C}}=1.402,1.401$ and $1.400 \AA$, respectively. In other words, the interaction between graphene and $\mathrm{SiO}_{2}$ substrate decreases as under $F \leq 0.02$ au, which agrees with $d_{0}(F=0)<d_{0}(F=0.01)<d_{0}(F=0.02)$ shown in Table $\mathbf{1}$.

The images of electron density difference of graphene/ $\mathrm{SiO}_{2}$ under different $F$ are shown in Fig. (2) where the red and the blue regions indicate the electron accumulation and the electron loss, respectively. In Fig. (2), both cations and anions contribute some charges of their own and share with each other, and charges mainly accumulate in the middle of a bond. Therefore, covalent bond for $\mathrm{SiO}_{2}$ is predominant although some amount of ionic bonding exists where the electron accumulation leans to $\mathrm{O}$ atoms, and $\mathrm{O}$ and $\mathrm{Si}$ atoms are separately charged negatively and positively. As $F$ increases, electrons accumulate in the interface region, and electron transfer to $\mathrm{C}$ atoms by the upward force due to $F$. At once $F=0.03$ au, more electrons pass through interface to $\mathrm{C}$ atoms overlayer and eventually covalent bonds between $\mathrm{C}$ and interface $\mathrm{O}$ are built up, which greatly affect the atomic structure and electronic density distribution, as shown in Figs. (1d, 2d).

It is well known that graphene on $\mathrm{SiO}_{2}$ substrate has excellent electronic transport properties [9,15,57]. In Figs. (2a, $\mathbf{2 b}$ and 2c), the interface with $F \leq 0.02$ au has a similar electronic density distribution where electrons lost on the both 
Table 2. Charges of Atoms in Graphene/ $\alpha-\mathrm{SiO}_{2}(0001)$ System as well as Charge Transfer $Q$ between $\mathrm{Graphene}$ and $\mathrm{SiO}(0001) \mathrm{Sub}$ strate Under Different $F$, Obtained by Mulliken Analysis. The Unit of the Atom Charge is one Electron Charge $e$, which is Elided Here for Clarity

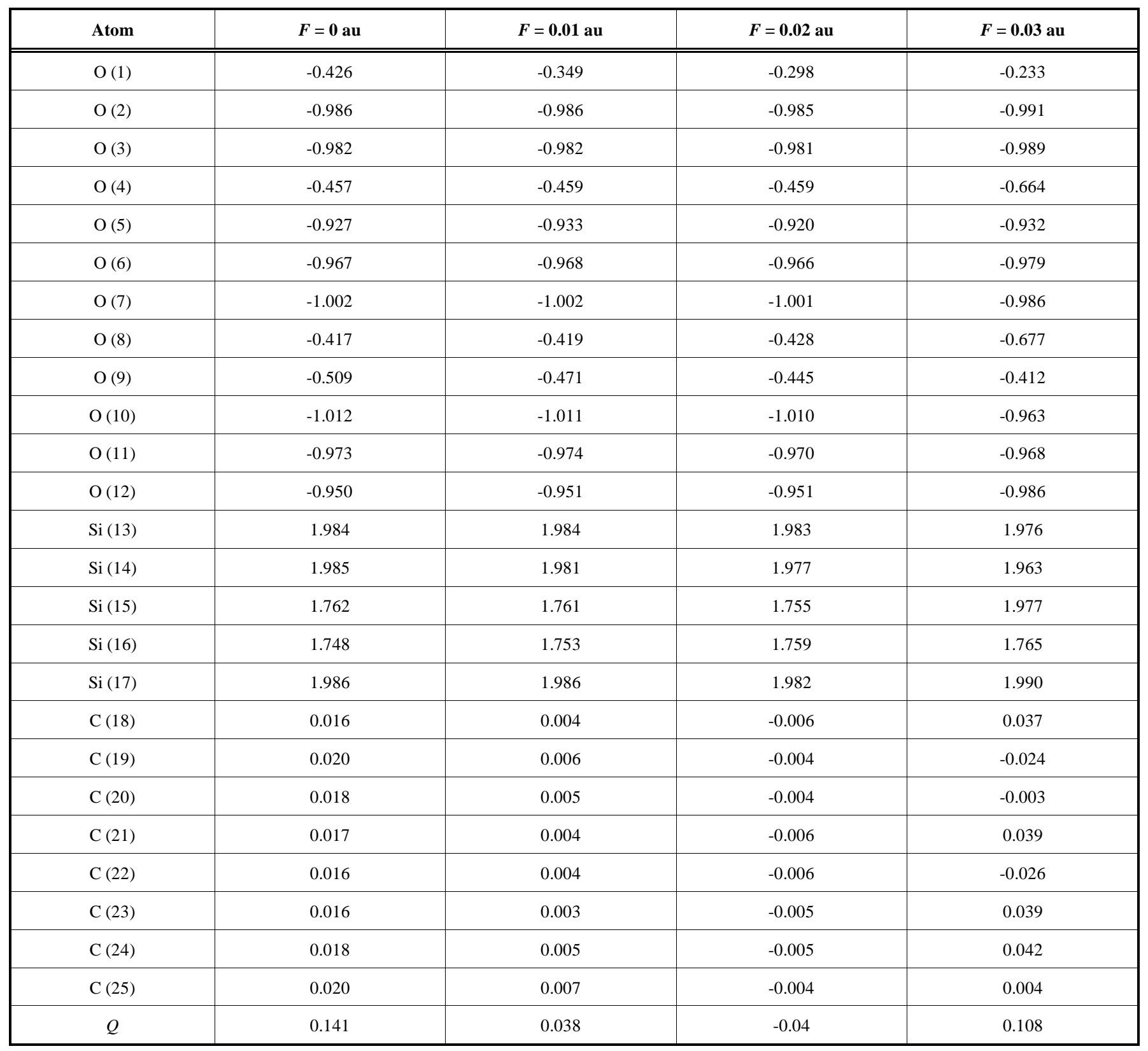

side of the $\mathrm{C}$ atomic layer and electrons accumulate in the center of the $\mathrm{C}$ layer where electrons are nearly averagely distributed. Thus, the electronic transport properties of the system under $F \leq 0.02$ au have slight changes. For the case of the system under $F=0.03$ au, electronic distribution in graphene is disordered due to the formation of $\mathrm{C}-\mathrm{O}$ bond. The gap of electronic distribution between graphene and the substrate disappears, and graphene integrates with $\mathrm{SiO}_{2}$ substrate together, as shown in Fig. (2d). As results, electronic transports in graphene are disturbed, which deteriorates the electronic transport properties of graphenes.

The above interpretations are supported by Mulliken analysis results, which are shown in Table $\mathbf{2}$ where charges of atoms in four different states are given. From Table $\mathbf{2}$, charges of $\mathrm{O} 4$ and $\mathrm{O} 8$ atoms in the interface of graphene $/ \mathrm{SiO}_{2}(0001)$ are respectively -0.457 and $-0.417 e$ un$\operatorname{der} F=0$. Their absolute values increase as $F$ increases, which are consistent with the result of Fig. (2) that electrons accumulate in the interface region as $F$ increases. For the structure with $F=0, \mathrm{C}$ atoms lose some electrons and have positive charge, and the lost electrons pass to $\mathrm{SiO}_{2}$ substrate. Meanwhile, the charges in graphene layer are inhomogeneously distributed. C19 and C25 have the largest charge values since they are nearest to interface $\mathrm{O}$ atoms, while $\mathrm{C} 18$, $\mathrm{C} 22$ and $\mathrm{C} 23$ located on hollow site of $\mathrm{O}$ atoms have the fewest charge. For the structure with $F=0.01$ and 0.02 au, atom location in graphene layer in parallel direction has 
slightly change due to similar charge distribution where C19 and $\mathrm{C} 25$ have the largest charge, and C18, C22 and C23 have the fewest charge. However, atom location in perpendicular direction varies evidently as charges of $\mathrm{C}$ atoms decrease remarkably where $d_{0}(F=0)<d_{0}(F=0.01)<$ $d_{0}(F=0.02)$.

The charge transfer $Q$ between graphene and $\mathrm{SiO}_{2}$ substrate under different $F$ is listed in Table 2. The electrons lost by $\mathrm{C}$ atoms become less as $F$ increases, and graphene even gets electrons from $\mathrm{SiO}_{2}$ substrate at $F=0.02$ au. This is because there is the upward force on electrons caused by the downward electric field. For the structure with $F=0.03$ au, the electronic density distributions of all atoms and atom locations in three dimensions are greatly changed and $Q$ becomes positive again due to the formation of strong $\mathrm{C}-\mathrm{O}$ bonds. As shown in Fig. (1d), C21 and C23 bind with $\mathrm{O} 8$ and $\mathrm{O} 4$ directly, and results in an uneven $\mathrm{C}$ monolayer. The two $\mathrm{C}$ atoms have $0.039 e$, while the two $\mathrm{O}$ atoms have -0.664 and $-0.677 e$, which lead to the drops of $d_{0}$ and that of the electron transfer from graphene layer to $\mathrm{SiO}_{2}$ substrate. Furthermore, it is found that band gap $E_{\mathrm{g}}$ of graphene changes with $F$ where $E_{\mathrm{g}}$ decreases from $0.26 \mathrm{eV}$ at $F=0$ to $0.16 \mathrm{eV}$ at $F=0.02 \mathrm{au}$, and jumps up to $0.41 \mathrm{eV}$ at $F=0.03$ au. This variation of $E_{\mathrm{g}}$ is due to the change of atomic structure shown in Fig. (1) induced by $F$. Namely, $E_{\mathrm{g}}\left(d_{0}=1.452\right)>$ $E_{\mathrm{g}}\left(d_{0}=3.227\right)>E_{\mathrm{g}}\left(d_{0}=3.273\right)>E_{\mathrm{g}}\left(d_{0}=3.587\right)$. The result is also consistent with the literature result that the decreasing of $d_{0}$ increases $E_{\mathrm{g}}$ of graphene [26]. Therefore, the electronic properties of graphene could be modulated by electric field, and large $F$ would destroy excellent electronic properties of graphene and should be avoided for applications.

\section{THE ADSORPTION PROPERTIES OF GRAPHENE}

\subsection{The Adsorption of CO Molecules In Intrinsic Gra- phene and Al-doped Graphene}

It was reported that the detectable range and sensitivity of the single wall carbon nanotubes (SWCNTs) can be widened and enhanced substantially through either doping technology or surface engineering [67-69]. For example, SWCNTs coated with $\mathrm{Pb}$ nanoparticles have high sensitivity to $\mathrm{H}_{2}$ [68], $\mathrm{SnO}_{2} / \mathrm{SWCNTs}$ hybrid material shows an enhanced sensitivity to $\mathrm{NO}_{2}$ [69]. The high sensitivity of boron doped SWCNT to $\mathrm{CO}$ and $\mathrm{H}_{2} \mathrm{O}$ absorptions has been also demonstrated [67]. Most recently, Al-cluster and Al-doped SWCNTs assembly are suggested to be promising systems for novel molecular sensors to $\mathrm{NH}_{3}$ [70] and $\mathrm{CO}$ [71], and the B doped SWCNTs are highly sensitive to the gaseous cyanide and formaldehyde molecules [72]. However, the devices with higher sensitivity to these toxic gases are apparently required for environmental safety issues both in workplaces and residential areas, especially in some industrial and military fields.

Graphene-based devices may be a solution for ultra-high sensitivity gas sensor [73-75]. Similar to CNTs, the working principle of graphene devices as gas sensors is based on the change of their electrical conductivity induced by surface adsorbates, which acts as either donors or acceptors associated with their chemical natures and preferential adsorption sites $[73,76,77]$. In this section, we report that the sensitivity of graphene system to $\mathrm{CO}$ gas could be enhanced to a higher level, which exceeds by orders of magnitudes state-of-the-art sensors, through Al doping.

All DFT calculations are performed in $\mathrm{Dmol}^{3}$ code $[62,78]$. It is widely known that calculations limited at the LDA overestimate bond energy $E_{\mathrm{b}}$ and underestimate equilibrium distances $[79,80]$. Thus, a GGA with the RPBE method is used as the exchange correlation function [63]. The DSPP core treatment [62] is implemented for relativistic effects, which replaces core electrons by a single effective potential. To ensure that the results of the calculations are comparable, identical conditions are employed for the isolated $\mathrm{CO}$ molecule, the clean Al-doped graphene and also the adsorbed graphene system. The $k$-point is set to $6 \times 6 \times 2$ for all slabs, which brings out the convergence tolerance of energy of $1.0 \times 10^{-5}$ hartree $(1$ hartree $=27.2114 \mathrm{eV})$, and that of maximum force of 0.002 hartree.

In the simulation, three-dimensional periodic boundary condition is taken and $\mathrm{C}-\mathrm{O}$ bond length is set to $l_{\mathrm{C}-\mathrm{O}}=1.13$ $\AA$, which is consistent with experimental results [81]. For graphene, a single layer $3 \times 3$ supercell with a vacuum width of $12 \AA$ above is constructed, which ensures that the interaction between repeated slabs in a direction normal to the surface is small enough. The variation of energetic results would be within a range of $0.1 \mathrm{eV}$ if the vacuum width is expanded from 12 to $15 \AA$. All atoms are allowed to relax for all energy calculations. The $E_{\mathrm{b}}$ between the $\mathrm{CO}$ gas molecule and graphene is defined as,

$$
E_{\mathrm{b}}=E_{\mathrm{CO}+\text { graphene }}-\left(E_{\text {graphene }}+E_{\mathrm{CO}}\right)
$$

where the subscripts $\mathrm{CO}+$ graphene, graphene, and $\mathrm{CO}$ denote the adsorbed system, isolated graphene and $\mathrm{CO}$ molecules, respectively.

To evaluate the interaction between a $\mathrm{CO}$ molecule and the intrinsic graphene or Al-doped graphene, $E_{\mathrm{b}}$ described in Eq. (10) and the binding distance, $d$, with all possible configurations are calculated. Twelve possible binding sites for the $\mathrm{CO}$ adsorbed on graphene layer are considered as initial structures. After full relaxation, no distinct structural change has been found. All of the results are displayed in Table 3. It is found that adsorption configuration shown Fig. (3f) has the smallest $d=3.768 \AA$ value and the largest $E_{\mathrm{b}}=0.016 \mathrm{eV}$ value among all possible adsorption configurations, or the most stable atomic arrangement, which are consistent with other simulation results of $E_{\mathrm{b}}=0.014 \mathrm{eV}$ and $d=3.740 \AA$ [74]. However, the $E_{\mathrm{b}}$ is still considered too small and $d$ too large although they are the most favorable one for adsorption, reflecting that $\mathrm{CO}$ undergoes weak physisorption on the intrinsic graphene, or the intrinsic graphene is insensitive to CO molecules.

When one carbon atom is substituted by $\mathrm{Al}$ atom in the super cell, the geometric structure of Al-doped graphene changes dramatically. Figs. $(\mathbf{4 a}, \mathbf{4 b})$ represent the geometries of intrinsic and Al-doped graphene after relaxation. As shown in Table 4 and Fig. (4b), the Al doping results in $l$ elongation from $l_{\mathrm{C}-\mathrm{C}}=1.420 \AA$ to $l_{\mathrm{Al}-\mathrm{C}}=1.632 \AA$. This is associated with the distortion of hexagonal structures adjacent to the larger $\mathrm{Al}$ atom, similar to the restructuring in Al-doped SWCNTs [71]. 
Table 3. Summary of Results for CO Adsorption on Intrinsic Graphene and Al-doped Graphene on Different Adsorption Sites. The Meaning of T, $B$ and $H$ are Given in the Caption of Fig. (3).

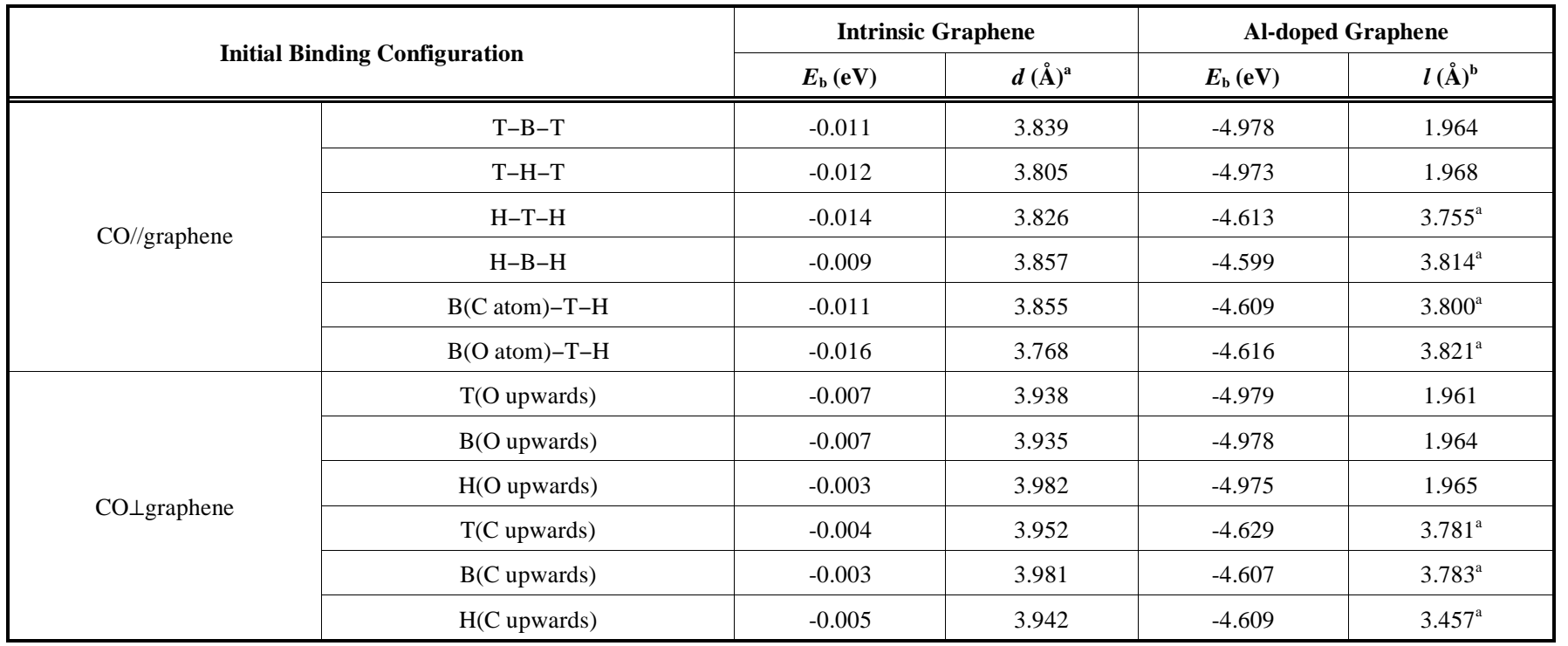

a. Binding distance between $\mathrm{CO}$ gas molecule and graphene layer.

b. Bond length of $\mathrm{Al}$ and $\mathrm{C}$ atom in $\mathrm{CO}$ gas molecule.

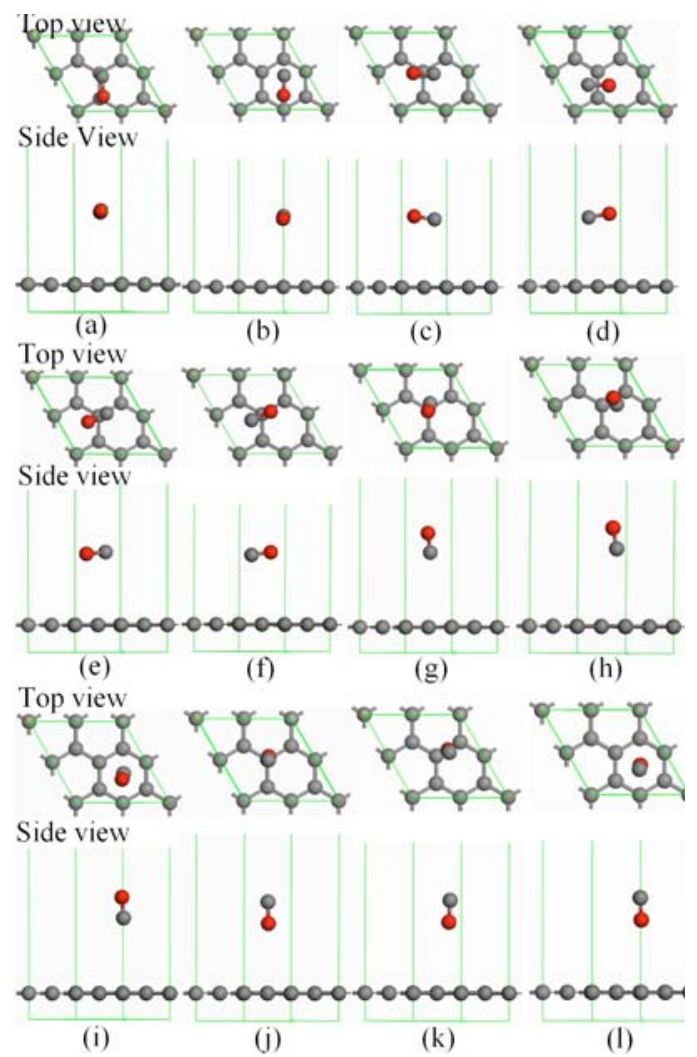

Fig. (3). Twelve available binding sites for $\mathrm{CO}$ adsorbed on intrinsic graphene (top and below images show the top and side view, respectively). (a) T-B-T, (b) T-H-T, (c) H-T-H, (d) H-B-H, (e) $\mathrm{B}(\mathrm{C}$ atom $)-\mathrm{T}-\mathrm{H}$, (f) $\mathrm{B}(\mathrm{O}$ atom $)-\mathrm{T}-\mathrm{H},(\mathbf{g}) \mathrm{T}(\mathrm{O}$ atom upward $),(\mathbf{h})$ $\mathrm{B}$ (O atom upward), (i) $\mathrm{H}$ (O atom upward), (j) T (C atom upward), (k) B (C atom upward), (1) $\mathrm{H}$ (C atom upward). T, B and $\mathrm{H}$ denote top site of $\mathrm{C}$ atoms, bridge site of $\mathrm{C}-\mathrm{C}$ bond and hollow site of carbon hexagon, respectively. Gray, pink and red spheres are denoted as $\mathrm{C}, \mathrm{Al}$ and $\mathrm{O}$ atoms, respectively. (Reproduced with permission from Ref. [28]. Copyright 2008, Elsevier).
When a $\mathrm{CO}$ molecule is adsorbed on Al-doped graphene, which one $\mathrm{C}$ atom substituted by an $\mathrm{Al}$ atom in the super cell, there are also twelve available adsorption sites similar to the case of $\mathrm{CO}$ absorption on intrinsic graphene shown in Fig. (3). These are taken as initial configurations. After relaxation, the configuration in Fig. (3d) has the most stable relaxed structure. Note that in Table $\mathbf{3}$, the deviation of $E_{\mathrm{b}}$ and $l_{\mathrm{Al}-\mathrm{C}}$ of the five configurations above are within the error of $1 \%$. The adsorption of $\mathrm{CO}$ causes a structure change in Al-doped graphene dramatically, resulting in an expansion of $l_{\mathrm{Al1}-\mathrm{C} 2}$ from 1.632 to $1.870 \AA$ while $l_{\mathrm{Al} 1-\mathrm{C} 4}$ elongates from 1.632 to $1.915 \AA$. The corresponding distance between the CO molecule and $\mathrm{Al}$ atom is $1.964 \AA$, being much shorter than 3.767 $\AA$ in the intrinsic graphene system. Moreover, the $E_{\mathrm{b}}$ of CO on Al-doped graphene is $4.979 \mathrm{eV}$, which is over 60 times larger than that of $\mathrm{CO}$ adsorbed on intrinsic graphene. Comparing with the $E_{\mathrm{b}}$ in other systems, such as $E_{\mathrm{b}}=1.280 \mathrm{eV}$ for CO/Al-doped SWCNT system [71], $E_{\mathrm{b}}=0.986 \mathrm{eV}$ for CO/B-doped SWCNT system [71] and $E_{\mathrm{b}}=0.201 \mathrm{eV}$ for $\mathrm{CO} / \mathrm{B}$-doped graphene etc, Al-doped graphene is energetically favorable for $\mathrm{CO}$ adsorption and is much more sensitive to the $\mathrm{CO}$ adsorption among the mentioned systems.

Furthermore, to investigate electronic structure changes of graphenes caused by $\mathrm{CO}$ adsorption, $Q$ values from either the intrinsic or Al-doped graphene to the polar CO molecules are calculated by Mulliken analysis, where $Q$ is defined as the charge variation caused by $\mathrm{CO}$ absorption. As listed in Table 4, $Q=0.027 e$ in Al-doped graphene is almost one order larger than $0.003 e$ in the intrinsic graphene. This supports the notion that $\mathrm{Al}$ doping influences the electronic properties of graphene substantially. This can also be verified by the difference of electronic densities between the intrinsic and Al-doped graphenes with and without the $\mathrm{CO}$ adsorption as shown in Fig. (5). In the figure, the red and blue regions represent the areas of electron accumulation and the electron loss, respectively. Fig. (5a) indicates that the 
Table 4. Some Structure Parameters of Intrinsic Graphene and Al-doped Graphene before and after Adsorption of CO Molecule

\begin{tabular}{|c|c|c|c|c|}
\hline System & Configuration & Bond & Bond length $l(\AA)$ & $Q(e)^{\mathrm{a}}$ \\
\hline \multirow[t]{6}{*}{ Intrinsic graphene } & Fig. (4a) & $\mathrm{C} 1-\mathrm{C} 2$ & 1.420 & \\
\hline & & $\mathrm{C} 1-\mathrm{C} 3$ & 1.420 & \\
\hline & & $\mathrm{C} 1-\mathrm{C} 4$ & 1.420 & \\
\hline & Fig. (4c) & $\mathrm{C} 1-\mathrm{C} 2$ & 1.420 & 0.003 \\
\hline & & $\mathrm{C} 1-\mathrm{C} 3$ & 1.421 & \\
\hline & & $\mathrm{C} 1-\mathrm{C} 4$ & 1.421 & \\
\hline \multirow[t]{6}{*}{ Al-doped graphene } & Fig. (4b) & $\mathrm{A} 11-\mathrm{C} 2$ & 1.632 & \\
\hline & & Al1-C3 & 1.632 & \\
\hline & & Al1-C4 & 1.632 & \\
\hline & Fig. (4d) & Al1-C2 & 1.870 & 0.027 \\
\hline & & Al1-C3 & 1.910 & \\
\hline & & Al1-C4 & 1.915 & \\
\hline
\end{tabular}

a. Electrons transferred from graphene layer to $\mathrm{CO}$ molecule. $e$ denotes one electronic charge.

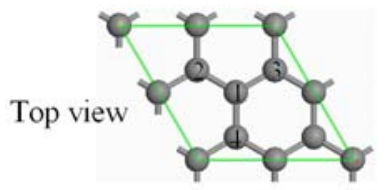

(a)

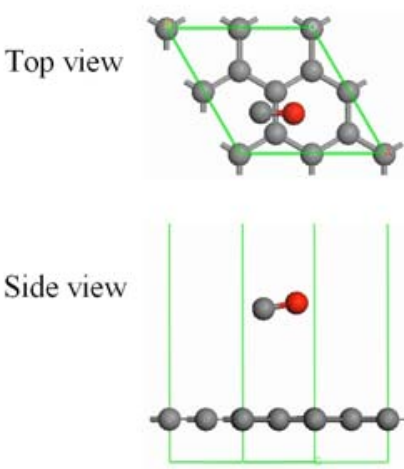

(c)

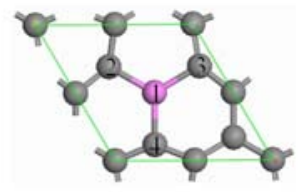

(b)

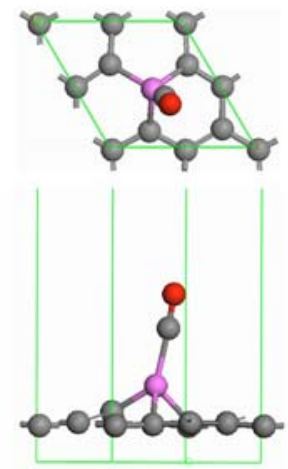

(d)
Fig. (4). Atomic configurations of intrinsic graphene and Al-doped graphene before and after adsorption of $\mathrm{CO}$ gas molecule where one $\mathrm{Al}$ atom dopes in site 1, and sites 2, 3 and 4 are $\mathrm{C}$ atoms near the doped Al atom. (a) and (b) are the relaxed configurations of intrinsic graphene and Al-doped graphene without adsorption. (c) and (d) are the preferred configurations after $\mathrm{CO}$ adsorption for intrinsic graphene and Al-doped graphene, respectively. (Reproduced with permission from Ref. [28]. Copyright 2008, Elsevier).

bond in the intrinsic graphene is of covalent nature because the preferential electron accumulation sites are mainly located within the bond rather than heavily centered on a particular atom. However, the Al doping modified the electron density by inducing the different electron affinities for Al and $\mathrm{C}$ atoms while the whole structure remains covalent in nature (Fig. (5b)). Physisorption of $\mathrm{CO}$ on the intrinsic gra- phene does not alter the electron distribution for both $\mathrm{CO}$ molecule and graphene, implying the weak bonding characteristics. It is discernable that electronic polarization is induced by the preferential accumulation of electrons on $\mathrm{O}$ in $\mathrm{CO}$ molecules (Fig. (5c)). As distinct from the $\mathrm{CO}$ absorption on the intrinsic graphene, the chemisorption of $\mathrm{CO}$ on Al-doped graphene leads to significant electron transfer from graphene to $\mathrm{CO}$ molecule (Fig. (5d)). In this case, the electrons not only accumulate on the $\mathrm{O}$ atom but also on the $\mathrm{C}$ atom of the molecule bond with the doped $\mathrm{Al}$ atom. The final position of $\mathrm{Al}$ atom in the chemisorbed $\mathrm{CO} / \mathrm{Al}$-graphene complex is thus a direct consequence of the maximized degree of $s p^{3}$ orbital hybridization with neighboring $\mathrm{C}$ atoms from both graphene layer and $\mathrm{CO}$ molecule. This is evidential because the red lobes around $\mathrm{C}$ atoms in Fig. (5d) are both pointing towards $\mathrm{Al}$ atom.

To further determine the effects of $\mathrm{CO}$ absorption on electrical conductivity, the DOS for both systems with and without the absorption are calculated. As shown in Figs. (6a, 6b), the Al doping in graphene enhances its electrical conductivity by shifting the highest DOS peak to just below the Fermi level $E_{\mathrm{f}}$, which also leads to decrease reduction of $E_{\mathrm{g}}$. This indicates that the doping $\mathrm{Al}$ atom induces shallow acceptor states in graphene like B atom in SWCNs, thus enhances its extrinsic conductivity [67]. When CO molecule adsorbed on the intrinsic and doped graphene surfaces, the total DOSs are shown in Figs. (4d, 6c). In the intrinsic graphene, the DOS of CO/graphene system near $E_{\mathrm{f}}$ has no distinct change, and the conductivity change is barely observable. It implies that the intrinsic graphene would not be an ideal CO gas sensor. However, for Al-doped graphene with the most stable chemisorbed CO configuration (Fig. (4d)), not only the highest DOS peak shifts over $E_{\mathrm{f}}$, but also the DOS value increases dramatically. This results in an $E_{\mathrm{g}}$ closure (Fig. (6d)) where $E_{\mathrm{g}}=0.18 \mathrm{eV}$ without adsorption and $E_{\mathrm{g}}=0$ with adsorption. It suggests that an extra number of 


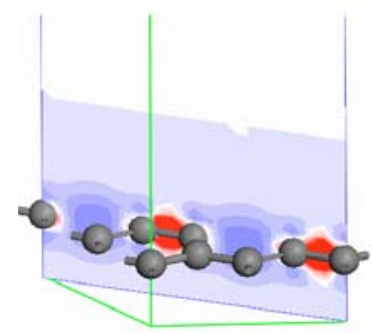

(a)

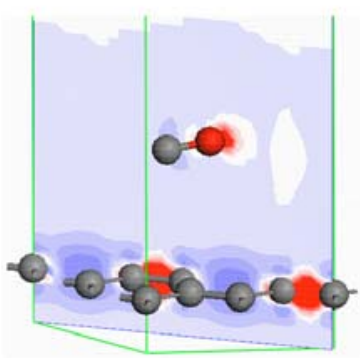

(c)

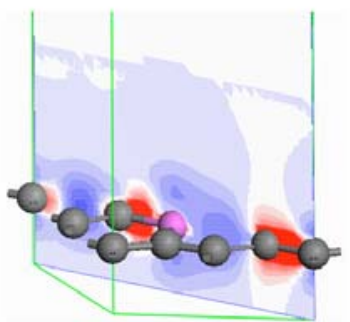

(b)

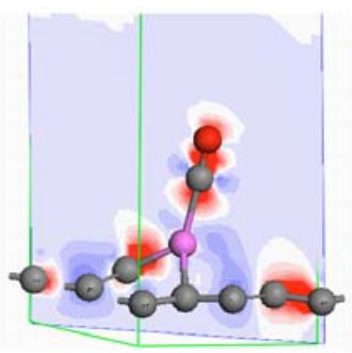

(d)
Fig. (5). Images of the electronic density difference for intrinsic graphene (a), Al-doped graphene (b), CO-graphene system with preferred configuration (c) and $\mathrm{CO}-\mathrm{Al}$-doped graphene system with preferred configuration (d). The red region shows the electron accumulation, while the blue region shows the electron loss. (Reproduced with permission from Ref. [28]. Copyright 2008, Elsevier).

shallow acceptor states is introduced when Al-doped graphene interacts with the highly polar $\mathrm{CO}$ molecule. As a result, the chemisorbed $\mathrm{CO}$ on Al-doped graphene will give rise to a large increase in the electrical conductivity of the doped graphene layer. By detecting the conductivity change of Al-doped graphene system before and after the adsorption of $\mathrm{CO}$, the presence of this toxic molecule can be detected sensitively. Therefore, Al-doped graphene is a promising sensor material for detecting $\mathrm{CO}$ molecules. However, desorption of $\mathrm{CO}$ molecule from Al-doped graphene is difficult due to the strong bonding of Al-CO [82]. This can be solved by applying $F$ to reactivate the sensor materials [83].

The favorable $\mathrm{CO}$ adsorption site on Al-doped graphene was identified through DFT calculations [28]. But it is still far to be optimized for actual applications. In particular, the effect of temperature $T$ on the adsorption/desorption behaviors on the $\mathrm{CO} /$ graphene system is still unclear.

Based on DFT results and thermodynamic analysis at $0 \mathrm{~K}$, the adsorption phase diagram can be established. The Gibbs free energy of adsorption $\Delta G_{\text {ads }}$ can be expressed as,

$$
\Delta G_{a d s}(T)=G_{a d s}(T)-G_{g}(T)-G_{C O}(T)
$$

where $G_{\text {ads }}(T), G_{\mathrm{g}}(T)$ and $G_{\mathrm{CO}}(T)$ are the corresponding Gibbs free energies of the adsorbed system, Al-doped graphene and the $\mathrm{CO}$ gas molecule at a particular $T$, respectively. Since the Gibbs free energy of the system before and after adsorption can be obtained with DFT results, the adsorption-desorption transition can be determined with Eq. (11) theoretically. The simulation method can be verified by tak-

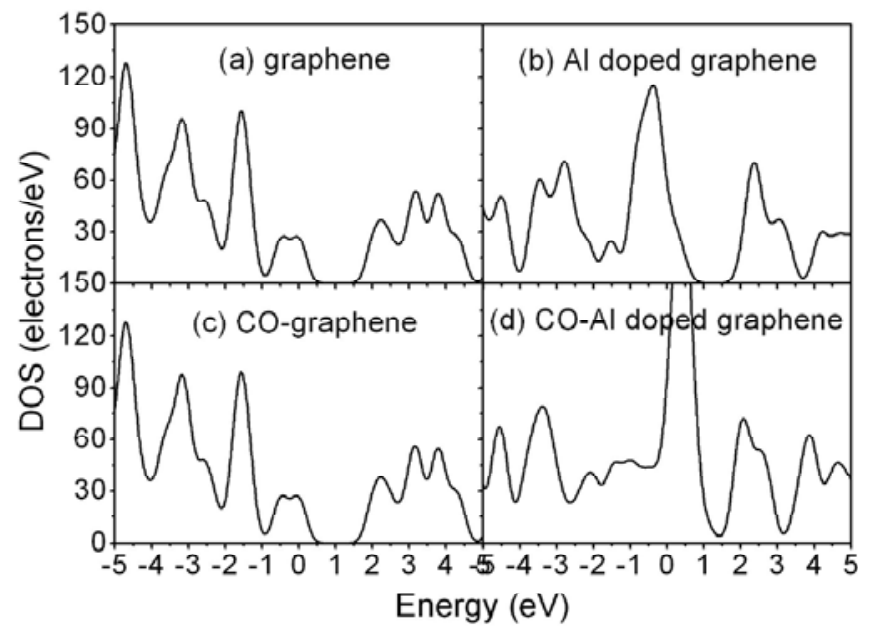

Fig. (6). Electronic density of state (DOS) of intrinsic graphene (a), Al-doped graphene (b), CO-graphene system with preferred configuration (c), and CO-Al-doped graphene system with preferred configuration (d). (Reproduced with permission from Ref. [28]. Copyright 2008, Elsevier).

ing the isolated $\mathrm{CO}$ molecule as a reference. The calculated results of bond length $l, E_{\mathrm{b}}$ and vibrational frequency $v_{\mathrm{C}-\mathrm{O}}$ of $\mathrm{C}-\mathrm{O}$ bond agree with the experimental [84] and theoretical data with two different potential functions [85] at $T=0$. The results show that $l_{\mathrm{C}-\mathrm{O}}, E_{\mathrm{b}}$ and $v_{\mathrm{C}-\mathrm{O}}$ are similar to experimental data within an error range of $0.7 \%$. Thus, the simulation method used in this work is applicable.

Subsequently, ab initio molecular dynamics (MD) calculations were performed under constant volume and constant temperature conditions (NVT) adopting GGA with the revised PBE method. The temperature effects on the atomic and electronic structures are calculated with a time step of 1 $f s$ at $300 \leq T \leq 450 \mathrm{~K}$ with an interval of $50 \mathrm{~K}$. The simulation time at the particular temperature is $2.5 \mathrm{ps}$ where the total energy fluctuates in the range of $0.01 \%$. MD is based on the velocity Verlet algorithm [86] for integration of the equation of motion. The implemented algorithm performs the Yoshida-Suzuki multiple-step numerical integration of varying quantity, depending on the choice of interpolation parameters $[87,88]$. A key parameter in the integration algorithms is the integration time step. A common rule-of-thumb used to set the time step is that the highest frequency vibration should be sampled between 10 and 20 times in one cycle. In this system, the frequency is in the order of $10^{13} \mathrm{~Hz}$, the time step is thus set as $1 f s$ within a reasonable range [89]. The temperature is controlled by algorithm of Nose [90]. The thermostate employs a feedback loop between the instantaneous kinetic energy and the set temperatures. The rate of feedback is determined by the mass parameter $q(q=2)$ [91-93].

With the thermal desorption method, $T$ dependent desorption time $\tau(T)$ can be expressed as [82,94]

$$
\tau(T)=v_{0}^{-1} e^{\left[-E_{a d s}(T) / K_{B} T\right]}
$$

where $K_{\mathrm{B}}$ is the Boltzmann's constant $\left(8.62 \times 10^{-5} \mathrm{eV} / \mathrm{K}\right)$, and $v_{0}$ is the attempt frequency of $10^{13} \mathrm{~Hz}$ for $\mathrm{CO}$ [89]. This 


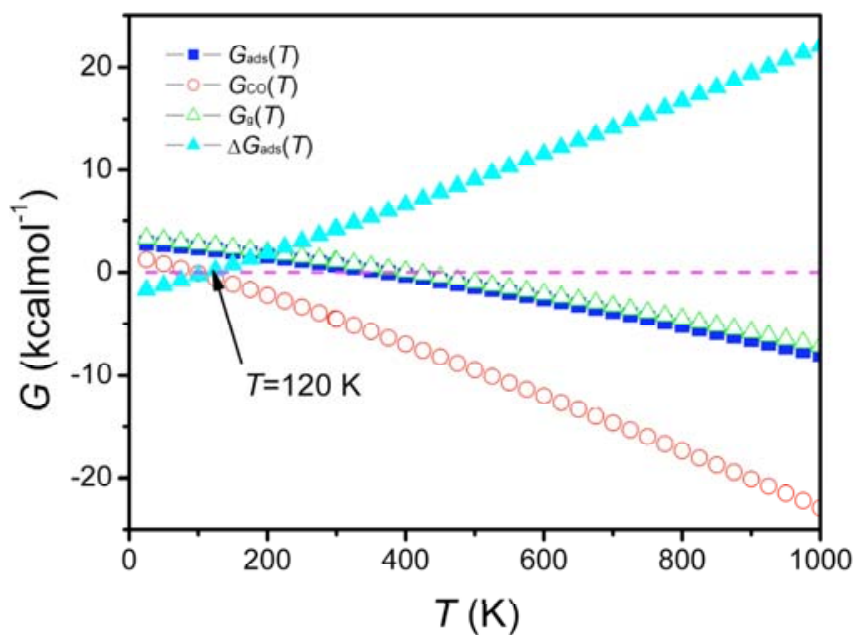

Fig. (7). The temperature dependent Gibbs free energy $G(T)$ functions where subscripts ads, g, and CO denote the adsorbed system, the isolated graphene and the $\mathrm{CO}$ molecules, respectively. And $\Delta G_{\text {ads }}(T)$ denotes Gibbs free adsorption energy, which is obtained in terms of eqn (2). The symbols are all DFT results. (Reproduced with permission from Ref. [37]. Copyright 2009, RSC).

thermal desorption method is close to the experimental conditions and it can be used to determine thermodynamic properties of the adsorption systems [94].

With the adsorption structures determined by DFT calculations at an ideal condition, the phase diagram of adsorption/desorption for the $\mathrm{CO}$ adsorbed on Al-doped graphene as a function of $T$ can be established with the atomistic thermodynamics described in Eq. (11). Such a simple approach allows the exploration of $\Delta G_{\text {ads }}$ in an actual condition with respect to experiments. $\Delta G_{\text {ads }}(T), G_{\text {ads }}(T), G_{\mathrm{g}}(T)$ and $G_{\mathrm{CO}}(T)$ functions are plotted in Fig. (7). The results show that $\Delta G_{\text {ads }}(T)$ increases as $T$ increases, and eventually becomes positive at $T_{\mathrm{d}}=120 \mathrm{~K}$ where $T_{\mathrm{d}}$ is defined as the desorption temperature, or the desorption of $\mathrm{CO}$ from Al-doped graphene occurs when $T>120 \mathrm{~K}$ at the ideal state with $\tau \rightarrow \infty$.

However, with $a b$ initio MD calculation at $T=300,350$, 400 and $450 \mathrm{~K}$ for $2.5 \mathrm{ps}$ to reach the equilibrium at each $T$, it is found that the desorption occurs at $450 \mathrm{~K}$. The atomic configurations at the different $T$ are shown in Fig. (8) and the corresponding atomic structure parameters are listed in Table 5. The results show that $T_{\mathrm{d}}$ is between 400 and $450 \mathrm{~K}$. Since both data for MD simulation and atomistic thermodynamics come from the simulation, the difference on $T_{\mathrm{d}}$ caused by the simulation methodologies is limited, which should be mainly induced by the short equilibrium time of $2.5 \mathrm{ps}$ used in MD simulation that is much shorter than the actual situation.

$E_{\text {ads }}(T)$ and $\tau(T)$ determined by Eqs. (10) and (12) are plotted in Figs. (9, 10), respectively. Fig. (9) shows that $E_{\text {ads }}(T)$ decreases linearly with increasing $T$. This is consistent with the classic Readhead formula [95]. In Fig. (10), $\tau(T)$ decreases exponentially with increasing $T$, showing $T_{\mathrm{d}} \approx$ $420 \mathrm{~K}$ at $\tau=2.5 \mathrm{ps}$. This is in agreement with the results obtained from MD simulation, in which desorption occurs at $400<T<450 \mathrm{~K}$. In the experimental environment, the optimal $\tau$ is in an order of microsecond ( $\mu \mathrm{s})$ [82], and $\tau(400 \mathrm{~K}) \approx$ $1 \mu$ s from Fig. (10), indicating that the gas sensor can be

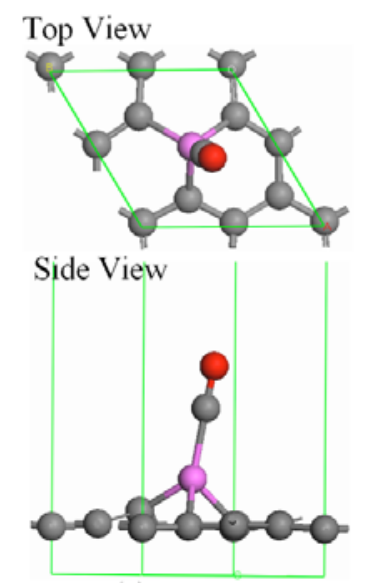

(a) $T=0$

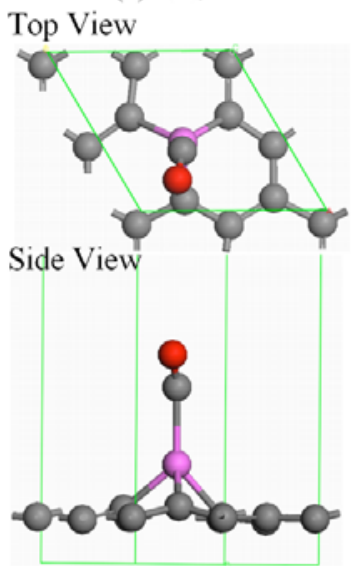

(c) $T=400 \mathrm{~K}$

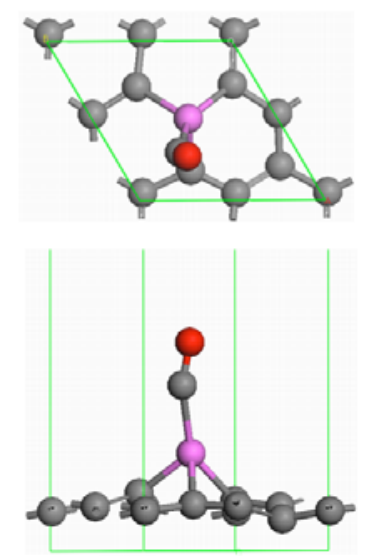

(b) $T=300 \mathrm{~K}$

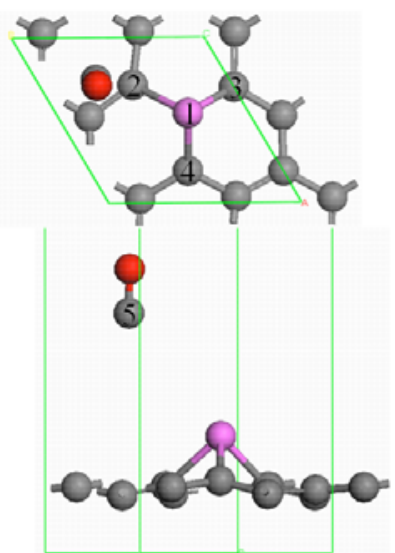

(d) $T=450 \mathrm{~K}$
Fig. (8). Atomic structure of CO molecule adsorption in Al-doped graphene at different temperature. For $T \neq 0$, the images are the configurations at simulation time $2.5 \mathrm{ps}$. In the figure, the uppermost atom is $\mathrm{O}$ atom, the third uppermost atom is $\mathrm{Al}$ atom, and the others are all $\mathrm{C}$ atoms. One $\mathrm{Al}$ atom dopes in site 1 and sites 2, 3 and 4 are $\mathrm{C}$ atoms near the doped $\mathrm{Al}$ atom, and site 5 is $\mathrm{C}$ atom in $\mathrm{CO}$ gas molecule. (Reproduced with permission from Ref. [37]. Copyright 2009, RSC).

reactivated for repeated applications by heating the materials up to $400 \mathrm{~K}$. Note that the adsorption-desorption process is dynamic. Once the CO molecule is adsorbed, the adsorption state would be remained for a time of $\tau$ until desorption occurs. During the adsorption period, electrical conductivity changes of Al-doped graphene can be detected.

Except for $T_{\mathrm{d}}$, the temperature dependence of atomic structures and electrical properties are also critical information for gas detection. Table $\mathbf{5}$ lists the structural parameters calculated by $a b$ inito $\mathrm{MD}$ at 300,400 and $450 \mathrm{~K}$, respectively. As $T$ increases, Al-CO bond length $l_{\mathrm{Al} 1-\mathrm{C} 5}$ increases, or the corresponding bond strength decreases. This is also evidenced by the $E_{\text {ads }}(T)$ declination as shown in Fig. (9). When $T$ further increases, desorption of $\mathrm{CO}$ from Al-doped graphene occurs (Fig. (8d)) where bond length of $l_{\text {Al1-C5 }}$ sharply changes from $2.097 \AA$ at $400 \mathrm{~K}$ to $4.590 \AA$ at $450 \mathrm{~K}$.

In order to better understand the results, Table 6 lists the charges of $\mathrm{C}$ atoms surrounding the doped $\mathrm{Al}$ atom, the doped $\mathrm{Al}$ atom and $\mathrm{CO}$ molecule as well as $Q$ between the 


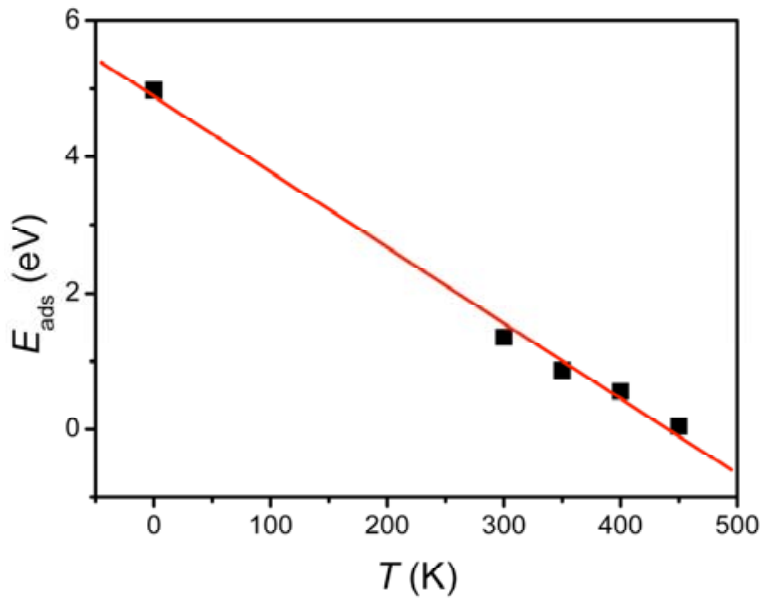

Fig. (9). Temperature dependent adsorption energy of CO molecule in Al-doped graphene $E_{\text {ads }}(T)$ function. The symbol $\boldsymbol{\square}$ is the MD simulation result at $T=0,300,350,400,450 \mathrm{~K}$. The solid line is the fitted linear function with the calculated data. (Reproduced with permission from Ref. [37]. Copyright 2009, RSC).

doped graphene and $\mathrm{CO}$ molecule, which are obtained by Mulliken analysis. $Q$ decreases as $T$ increases and $\mathrm{Al}$ atom loses electrons. The negative charge of the $\mathrm{C}$ atoms surrounding the doped $\mathrm{Al}$ also decreases. It results in that the charge difference between $\mathrm{C}$ and $\mathrm{Al}$ atoms decreases and the Al-C bond length in graphene layer elongates as $T$ increases. This is consistent with the structure parameter change listed in Table 5. On the other hand, the electrons in $\mathrm{C} 5$ and $\mathrm{O} 6$ of CO molecule also decrease with increasing $T$, and even C5 is positive at $T=450 \mathrm{~K}$. Due to the static interaction, $l_{\mathrm{C} 5-\mathrm{O} 6}$

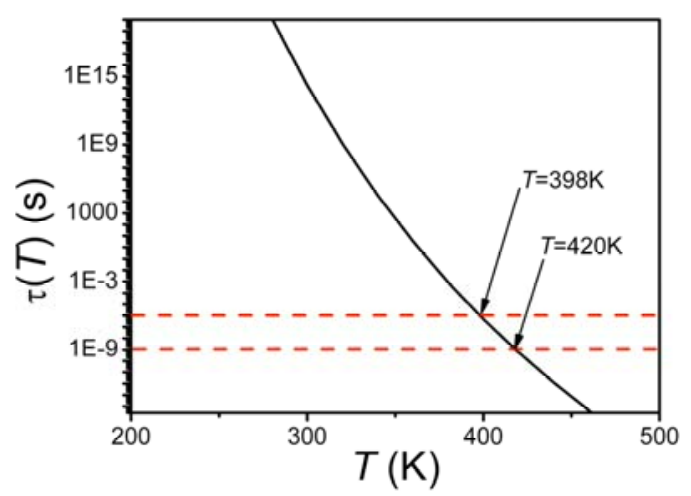

Fig. (10). Temperature dependent desorption time function $\tau(T)$ in terms of Eq. (3) where $E_{\text {ads }}(T)$ function needed is from Fig. (10). The two temperature 398 and $420 \mathrm{~K}$ are corresponding desorption temperature in MD simulation and actual situation. (Reproduced with permission from Ref. [37]. Copyright 2009, RSC).

decreases as $T$ increases as shown in Table $\mathbf{5}$.

The electronic density differences at various $T$ are calculated to further analyze charge distribution as shown in Fig. (11). It indicates that the bond of the $\mathrm{CO} /$ graphene system is covalent because the preferential electrons mainly accumulate in the bond rather than in a particular atom. However, due to different electronegativities of $\mathrm{C}, \mathrm{Al}$ and $\mathrm{O}$ atoms, electrons lean to the $\mathrm{C}$ atom for A11-C5 bond and $\mathrm{O}$ atom for the C5-O6 bond. Furthermore, with increasing $T$, electron transfer from the doped graphene to $\mathrm{CO}$ molecule is limited. This is evidenced by the smaller and thinner red regions around $\mathrm{CO}$ molecule in Fig. (11).

Table 5. Some Structure Parameters of CO Molecule Adsorbed on Al-Doped Graphene at Different Temperature, where $l$ is Bond Length

\begin{tabular}{|c|c|c|c|c|}
\hline & $\boldsymbol{T = 0}$ & $\boldsymbol{T = 3 0 0 ~ K}$ & $\boldsymbol{T = 4 0 0 ~ K}$ & $\boldsymbol{T}=\mathbf{4 5 0} \mathbf{K}$ \\
\hline \hline$l_{\mathrm{A} 1-\mathrm{C} 2}(\AA)$ & 1.872 & 1.880 & 1.946 & 1.973 \\
\hline$l_{\mathrm{A} 1-\mathrm{C} 3}(\AA)$ & 1.910 & 1.961 & 1.972 & 1.993 \\
\hline$l_{\mathrm{A} 1-\mathrm{C} 4}(\AA)$ & 1.916 & 1.923 & 1.929 & 1.989 \\
\hline$l_{\mathrm{A} 11-\mathrm{C} 5}(\AA)$ & 1.964 & 1.982 & 2.097 & 4.590 \\
\hline$l_{\mathrm{C} 5-\mathrm{O}}(\AA)$ & 1.164 & 1.161 & 1.159 & 1.157 \\
\hline
\end{tabular}

Table 6. Charges of Atoms Surrounding Doped Al Atom and Doped Al atom, and Changes Transferred $Q$ form Graphene to CO Gas Molecule at Different Temperature

\begin{tabular}{|l|c|c|c|c|}
\hline & $\boldsymbol{T = 0}$ & $\boldsymbol{T = 3 0 0 ~ K}$ & $\boldsymbol{T = 4 0 0 ~ K}$ & $\boldsymbol{T}=\mathbf{4 5 0} \mathbf{K}$ \\
\hline \hline A11 & 0.896 & 0.833 & 0.765 & 0.613 \\
\hline C2 & -0.363 & -0.303 & -0.324 & -0.274 \\
\hline C3 & -0.275 & -0.287 & -0.245 & -0.276 \\
\hline C4 & -0.267 & -0.239 & -0.255 & -0.216 \\
\hline C5 & -0.101 & -0.089 & -0.029 & 0.111 \\
\hline O & -0.128 & -0.132 & -0.127 & -0.123 \\
\hline$Q$ & 0.229 & 0.221 & 0.156 & 0.012 \\
\hline
\end{tabular}




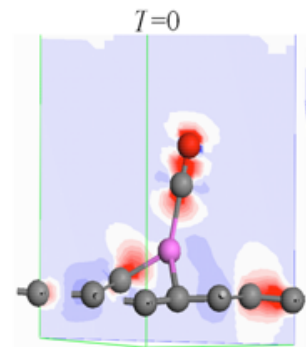

(a)

$T=400 \mathrm{~K}$

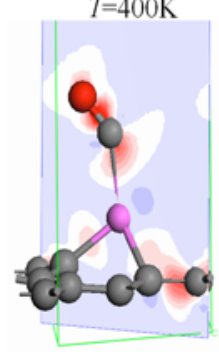

(c)

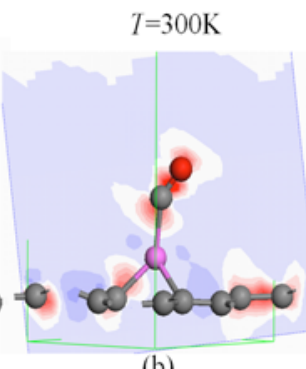

(b)

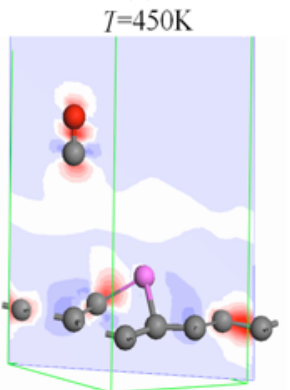

(d)
Fig. (11). Images of the electronic density difference of CO/graphene system at $T=0$ (a), $T=300 \mathrm{~K}$ (b), $T=400 \mathrm{~K}$ (c) and $T=450 \mathrm{~K}(\mathrm{~d})$. The red region shows the electron accumulation, while the blue region shows the electron loss. (Reproduced with permission from Ref. [37]. Copyright 2009, RSC).

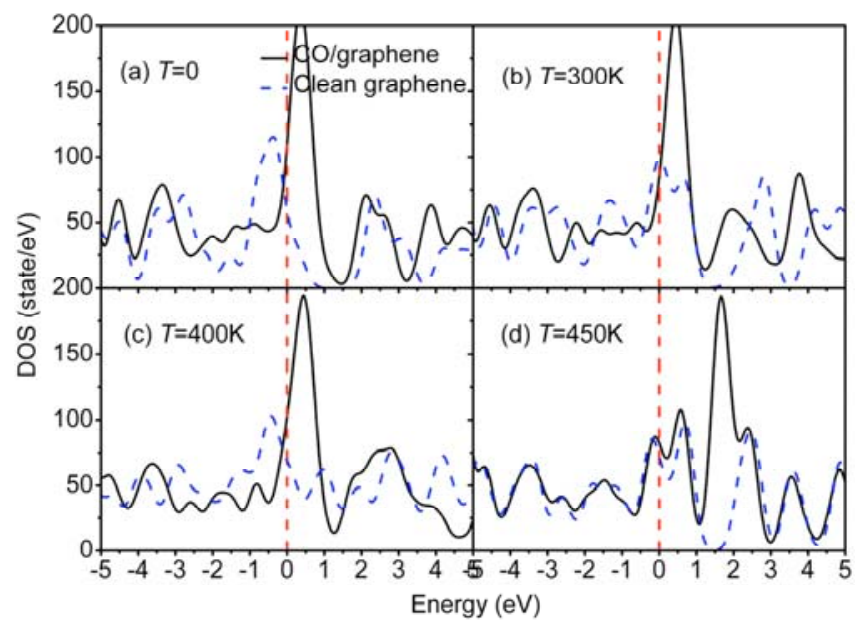

Fig. (12). Electronic density of state (DOS) of CO/graphene system at $T=0$ (a), $T=300 \mathrm{~K}$ (b), $T=400 \mathrm{~K}$ (c) and $T=450 \mathrm{~K}$ (d). The dash lines denote the Fermi energy location and the dash curves are the DOS of Al-doped graphene. (Reproduced with permission from Ref. [37]. Copyright 2009, RSC).

To understand the effect of $T$ on conductivity changes with and without the adsorption, the temperature dependence of DOS for Al-doped graphene and CO/graphene system are shown in Fig. (12). From Landauer formula [96], the number of bands crossing $E_{\mathrm{f}}$ determines the number of conductional channels or the conductivity of CO/graphene system [97,98]. Following this consideration, the largest conductivity change induced by the adsorption with suitable $\tau(T)$ value is found at $T=400 \mathrm{~K}$ where the best performance of $\mathrm{CO}$ detection is realized.

Although the adsorption/desorption time of an individual $\mathrm{CO}$ molecule at a particular position of Al-doped graphene has been investigated above, the response time for the actual sensor that involve a number of $\mathrm{CO}$ molecules in a certain period still retains unclear. It is believed that the actual response time, which is associated with a complex gas adsorption/desorption processing and sensitive to the flow rate and gas concentration, could be described with the statistical kinetics [99]. It needs to be investigated with other methodologies including experiments. This is not in the scope of this work but it will be implemented in future.

\subsection{Hydrogen Storage in Graphene Based Materials}

In recent years, hydrogen-based fuel systems have been considered to be a highly important topic of research for future energy schemes as hydrogen is a more efficient fuel in comparison to the existing carbonaceous fossil fuels [100,101]. Despite many recent technological developments in the hydrogen-based fuel systems, it is still an enormous challenge to have safe and efficient reversible hydrogen storage systems at ambient conditions [101]. One possible way for hydrogen storage is an efficient and controllable adsorption/desorption system. Carbon based materials appears promising for such a purpose. Several mechanisms of hydrogen storage through both physisorption and chemisorption have been proposed [102-106]. However, most of these efforts are far to reach the target of $6 \mathrm{wt} \%$ and binding strength of $-0.20 \sim-0.70 \mathrm{eV} / \mathrm{H}_{2}$ at ambient temperature and modest pressure for commercial applications specified by U.S. Department of Energy (DOE).

With DFT simulations, it was predicted that a single ethylene molecule can form a stable complex with two transition metals, thus adsorbing ten $\mathrm{H}_{2}$ molecules and lead to a high storage capacity of $\sim 14 \mathrm{wt} \%$ [107]. In addition, the highest $\mathrm{H}_{2}$ storage capacity of $13 \mathrm{wt} \%$ in a fullerene cage with twelve $\mathrm{Li}$ atoms capped onto the pentagonal faces was calculated [108]. This system has average adsorption energy $E_{\mathrm{b}}=-0.075 \mathrm{eV} / \mathrm{H}_{2}$. However, all DFT results are in the ideal condition at $T=0 \mathrm{~K}$, their performances at the DOE specified operation conditions are unclear.

Since carbon nanostructures have high surface areas, and thermal stability along with unique mechanical properties, improvement of their adsorption capacity by suitable modification would be of immense interest [102-108]. Thus, hydrogen storage using carbon nanostructures is still an important issue and deserves more attention. In this section, the potential of Al-doped graphene as hydrogen storage materials is investigated. The advantages of graphene are: (1) a large surface for hydrogen adsorption, (2) economical and scalable production [21], and (3) the strongest material ever measured [40].

$\mathrm{AlH}_{3}$ and related aluminum hydrides as hydrogen storage materials have recently become the focus of renewed interest $[109,110]$ as their potentially large hydrogen capacity of $\sim 10$ $\mathrm{wt} \%$. These materials are thermodynamically unstable in ambient temperature, but it is kinetically stable without much lost of hydrogen for years. Despite these excellent properties, extremely high pressure (exceeding $2.5 \mathrm{GPa}$ ) is required for hydrogen adsorption. While these hydrides possess a small negative enthalpy of formation [110], the large hydrogen desorption energy proves impractical for practical applications. The origin of this energy barrier lies in the 
rather strong mixed ionic and covalent bonds [110] formed between $\mathrm{Al}$ and $\mathrm{H}$. Thus it is essential to significantly reduce the desorption energy.

There appears another way for $\mathrm{Al}$ atoms to store hydrogen, i.e., to further decrease the interaction between $\mathrm{Al}$ and $\mathrm{H}$. In this way, the weak chemisorption can be changed into strong physisorption. For hydrogen storage through physisorption, strong interaction between $\mathrm{H}_{2}$ molecules and a large surface area for adsorption is required. The unique characteristics of graphene and $\mathrm{Al}$ for hydrogen storage lead to an investigation of the properties of Al-doped graphene as a possible hydrogen storage candidate. It would be intriguing to understand the interaction between graphene, $\mathrm{Al}$ and $\mathrm{H}$. In this work, the adsorption behavior of $\mathrm{H}_{2}$ in Al-doped graphene was studied by DFT calculation. In addition, we processed the $a b$ initio MD calculation to investigate the effects of $T$ and $P$ on adsorption/desorption behaviors of this system.

All DFT calculations are performed in $\mathrm{Dmol}^{3}$ code [78]. Previous studies $[111,112]$ have shown that the LDA prediction of the physisorption energies of $\mathrm{H}_{2}$ on the surface of graphite and carbon nanotubes are in good agreement with experiments. The reliability of LDA can be ascribed to the following facts [111]: (1) When the electron densities of $\mathrm{H}_{2}$ and graphene overlap weakly, the nonlinearity of the exchange-correlation energy density functional produces an attractive interaction even in the absence of electron density redistribution; (2) The overestimated binding energy by LDA $[80,74]$ may compensate for the insufficient account of van der Waals interactions [111]. In contrast, DFT calculation using a GGA produced a purely repulsive interaction. Using a GGA-PW91 functional, a repulsive interaction between $\mathrm{H}_{2}$ and a graphene layer and also between $\mathrm{H}_{2}$ and a $(6,6)$ carbon nanotube was obtained [113]. This contradicts the experimental findings [114]. It was noted that LDA calculations well reproduce the empirical interaction potentials between graphitic layers and also in the other graphitic systems for distances near to the equilibrium separation although the LDA is not able to reproduce the long-range dispersion interaction [115]. Therefore, LDA is selected in this work. To ensure that the calculated results are comparable, identical conditions are employed for the isolated $\mathrm{H}_{2}$ molecules and graphene, and also the adsorbed graphene system. The $k$-point is set to $6 \times 6 \times 2$ for all slabs, which brings out the convergence tolerance of energy of $1.0 \times 10^{-5}$ hartree (1 hartree $=27.2114 \mathrm{eV}$ ), and that of maximum force is 0.002 hartree/A.

In the simulation, three-dimensional periodic boundary condition is taken and $\mathrm{H}-\mathrm{H}$ bond length is set to $l_{\mathrm{H}-\mathrm{H}}=0.74$ $\AA$, which is consistent with experimental results [81]. Graphene used in our simulation consist of a single layer of $3 \times 3$ supercell with a vacuum width of $12 \AA$ to minimize the interlayer interaction. Increasing the vacuum width will greatly increase the computation expense although it has only negligible consequence on the results obtained. All atoms are allowed to relax in all energy calculations. The adsorption energy $E_{\mathrm{b}}$ between the $\mathrm{H}_{2}$ gas molecule and graphene is defined as,

$$
E_{\mathrm{b}}=E_{\mathrm{H} 2+\text { graphene }}-\left(E_{\text {graphene }}+E_{\mathrm{H} 2}\right)
$$

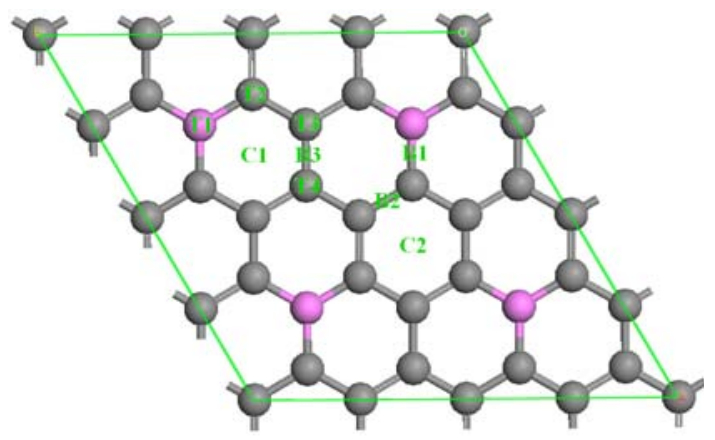

Fig. (13). Eight different adsorption sites on Al-doped graphene. The gray and pink balls are respectively $\mathrm{C}$ and $\mathrm{Al}$ atoms. (Reproduced with permission from Ref. [29]. Copyright 2009, AIP).

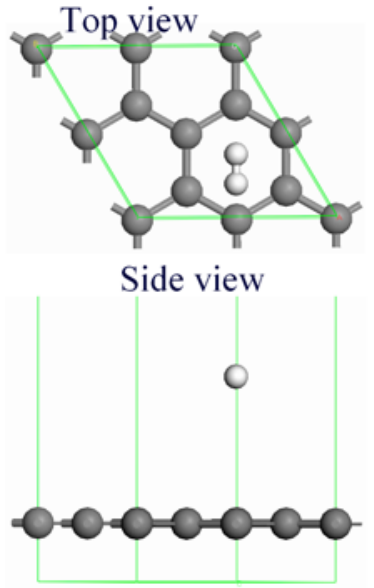

(a)
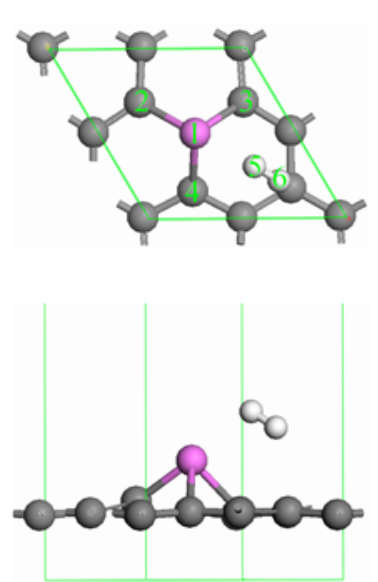

(b)
Fig. (14). The favorite adsorption configurations with $1 \mathrm{H}_{2}$ molecule adsorbed in intrinsic graphene (a), and in Al-doped graphene (b). The white balls are $\mathrm{H}$ atoms. (Reproduced with permission from Ref. [29]. Copyright 2009, AIP).

where the subscripts $\mathrm{H}_{2}$ +graphene, graphene, and $\mathrm{H}_{2}$ denote the adsorbed system, isolated graphene and $\mathrm{H}_{2}$ molecules, respectively.

For Al-doped graphene, the concentration of $\mathrm{Al}$ is 12.5 at $\%$ with the additional constrain that there is only one $\mathrm{Al}$ atom per graphene hexagonal ring (Fig. (13)) to avoid $\mathrm{Al}$ atoms clustering on graphene [116]. For $\mathrm{H}_{2}$ adsorption on Al-doped graphene, there are 4 top sites of T1, T2, T3 and $\mathrm{T} 4$, and 3 bridge sites of $\mathrm{B} 1, \mathrm{~B} 2$ and $\mathrm{B} 3$, and 2 center sites of $\mathrm{C} 1$ and $\mathrm{C} 2$, as shown in Fig. (13). (In this figure, a larger simulation cell is given in order to better display the different adsorption sites on Al-doped graphene. Fig. (14) reflects the actual simulation cell size.) At each adsorption site, there are two highly symmetrical adsorption configurations, namely $\mathrm{H}_{2}$ molecule resides parallel or perpendicular to graphene surface. Therefore, a total of 18 adsorption configurations for $\mathrm{H}_{2}$ on Al-doped graphene are present.

Due to the periodicity of $\mathrm{H}_{2}$ adsorbed in intrinsic graphene or Al-doped graphene systems, we have selected the unit cell with the following conditions: eight $\mathrm{C}$ atoms and one $\mathrm{H}_{2}$, or seven $\mathrm{C}$ atoms, one $\mathrm{Al}$ atom and one $\mathrm{H}_{2}$ (see Fig. (14)). If we place a $\mathrm{H}_{2}$ at any location of the cell, the distance 
Table 7. Summary of Results for $\mathrm{H}_{2}$ adsorption on Intrinsic graphene and Al-doped Graphene on Different Adsorption Sites. For $\mathrm{H}_{2}$ Adsorption on Intrinsic Graphene, there are 6 Different Adsorption Sites as Listed in the Table. For $\mathbf{H}_{2}$ Adsorption on Al-doped Graphene, there are 18 Different Adsorption Configurations as Shown in Fig. (13).

\begin{tabular}{|c|c|c|c|c|c|c|}
\hline \multirow{2}{*}{\multicolumn{2}{|c|}{ Initial Binding Configuration }} & \multicolumn{2}{|c|}{ Intrinsic Graphene } & \multicolumn{3}{|c|}{ Al-Doped Graphene } \\
\hline & & \multirow{2}{*}{$\begin{array}{r}\boldsymbol{E}_{\mathrm{b}}(\mathrm{eV}) \\
-0.136\end{array}$} & \multirow{2}{*}{$\frac{\boldsymbol{d}(\mathbf{(})^{\mathrm{b}}}{2.845}$} & \multirow{2}{*}{$\begin{array}{r}\boldsymbol{E}_{\mathbf{b}}(\mathbf{e V}) \\
-0.209\end{array}$} & \multirow{2}{*}{$\frac{l(\AA)^{\mathrm{a}}}{2.762}$} & \multirow[t]{2}{*}{$d(\AA)^{\mathrm{b}}$} \\
\hline \multirow{8}{*}{$\mathrm{H}_{2} / /$ graphene } & $\mathrm{T} 1$ & & & & & \\
\hline & $\mathrm{T} 2$ & & & -0.34 & 2.526 & 2.682 \\
\hline & $\mathrm{T} 3$ & & & -0.407 & 2.588 & 2.486 \\
\hline & $\mathrm{T} 4$ & & & -0.361 & 2.942 & 2.537 \\
\hline & B2 & & & -0.411 & 2.527 & 2.575 \\
\hline & B3 & & & -0.411 & 2.506 & 2.563 \\
\hline & $\mathrm{C} 1$ & -0.159 & 2.635 & -0.427 & 2.083 & 2.073 \\
\hline & $\mathrm{C} 2$ & & & -0.188 & & 2.657 \\
\hline \multirow{6}{*}{$\mathrm{H}_{2} \perp$ graphene } & $\mathrm{T} 4$ & & & -0.33 & 2.976 & 2.179 \\
\hline & B1 & -0.142 & 2.620 & -0.206 & 2.271 & 3.732 \\
\hline & $\mathrm{B} 2$ & & & -0.412 & 2.468 & 2.595 \\
\hline & B3 & & & -0.426 & 3.196 & 2.074 \\
\hline & $\mathrm{C} 1$ & -0.148 & 2.425 & -0.426 & 2.092 & 2.104 \\
\hline & $\mathrm{C} 2$ & & & -0.24 & 3.117 & 2.468 \\
\hline
\end{tabular}

a. Distance between $\mathrm{Al}$ and $\mathrm{H}_{2}$.

b. Distance between $\mathrm{H}_{2}$ molecule and graphene layer.

from this $\mathrm{H}_{2}$ to other $\mathrm{H}_{2}$ molecules in the nearest cells is $4.920 \AA$. This large separation, compared to the bond length of $\mathrm{H}_{2}(0.740 \AA)$, would ensure that there is no interaction between $\mathrm{H}_{2}$ molecules in different cells [117].

To calculate $\mathrm{H}_{2}$ adsorption capability of Al-doped graphene at room temperature and modest pressure, we performed ab initio MD calculation with CASTEP (Cambridge Sequential Total Energy Package) code based on the structure obtained by DFT above, which utilizes plane-wave pseudopotential to perform the first principle quantum mechanics calculations [118]. LDA with the Ceperley-AlderPerdew-Zunger (CAPZ) function $[119,120]$ was employed as exchange-correlation functions, cutoff energy $E_{\mathrm{c}}=280 \mathrm{eV}$ and $k$-points is $6 \times 6 \times 2$. In principle, $E_{\mathrm{c}}$ increases until the calculated total energy converges within the required tolerance. $d E_{\text {tot }} / d \ln E_{\mathrm{c}}$ is the parameter used to evaluate the accuracy of the calculation, where $E_{\text {tot }}$ is the total energy of the system. The software can calculate $d E_{\text {tot }} / d \ln E_{\mathrm{c}}$ value automatically. In general, $d E_{\mathrm{tot}} / d \ln E_{\mathrm{c}}=0.1 \mathrm{eV} /$ atom is sufficient for the most calculations. The values of $k$-points are increased until the calculated energy converges within the required tolerance, where the $k$-points sample the irreducible wedge of the Brillouin zone. In this work, the $k$-points of $6 \times 6 \times 2$ for all slabs have the energy convergence tolerance of $1.0 \times 10^{-6} \mathrm{eV} /$ atom. Such energy tolerance is small enough to ensure establishment of the actual equilibrium structure.
Each MD simulation was performed in NPT statistical ensemble, i.e. constant numbers of atoms $N$, pressure $P$ and $T$, with $T=300 \mathrm{~K}$ and $P=0.0001 \sim 1 \mathrm{GPa}$. Time step of $1 \mathrm{fs}$ was selected and simulation time at a particular $T$ was $2.5 \mathrm{ps}$ where the total energy fluctuation was in the range of $0.01 \%$. The same was selected for $\mathrm{H}_{2} \mathrm{~S}$ dissociation on the $\mathrm{Fe}(110)$ surface [91]. A Verlet algorithm [86] was used to integrate the equations of motion, with $T$ controlled by algorithm of Nose [121], and $P$ was controlled according to the Parrinello-Raham algorithm [122]. A key parameter in the integration algorithms is the integration time step. A common rule-of-thumb used to set the time step is that the highest frequency vibration should be sampled between 10 and 20 times in one cycle. In this system, the frequency is in the order of $10^{13} \mathrm{~Hz}$ [123], the time step is thus set as $1 f s$ within a reasonable range.

After geometry relaxation, $E_{\mathrm{b}}$ values and the corresponding structure parameters of the 18 adsorption configurations for $\mathrm{H}_{2}$ adsorbed in the intrinsic graphene are listed in Table 7. It was found that the most favorable configuration is $\mathrm{H}_{2}$ adsorbed on the center site of the carbon ring with $E_{\mathrm{b}}=-0.159$ $\mathrm{eV}$ as shown in Fig. (14a) and the distance between $\mathrm{H}_{2}$ and graphene $d=2.635 \AA$. The results are consistent with other reported results of $E_{\mathrm{b}}=-0.133 \mathrm{eV}$ and $d \approx 2.8 \AA$ [112]. The small magnitude of $E_{\mathrm{b}}(<0.1 \mathrm{eV})$ shows that the system is in the weak physisorption region. It indicates that the intrinsic graphene is not suitable for hydrogen storage. 
(a) $\mathrm{H}_{2}$ /graphene

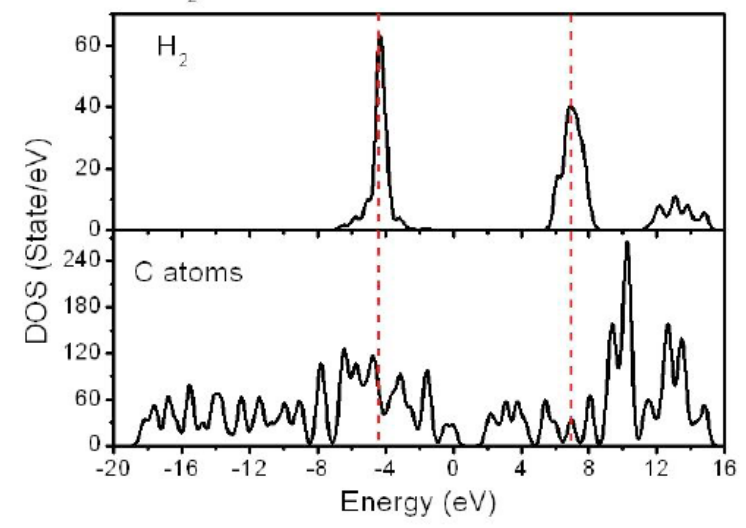

(b) $\mathrm{H}_{2}$ /Al-doped-graphene

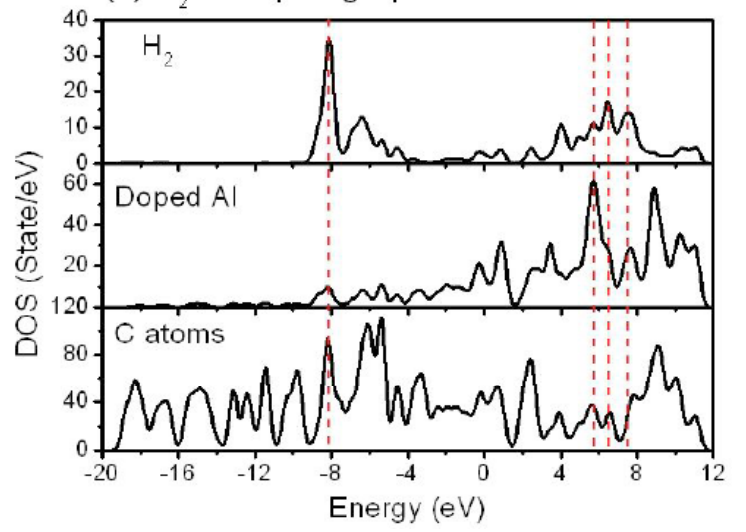

Fig. (15). Electronic density of states (DOSs) of adsorbed $\mathrm{H}_{2}$, doped $\mathrm{Al}$ and graphene for both the $\mathrm{H}_{2}$ /graphene and $\mathrm{H}_{2}$ /Al-doped-graphene systems as shown in panel (a) and panel (b), respectively. (Reproduced with permission from Ref. [29]. Copyright 2009, AIP).

For the adsorption of $\mathrm{H}_{2}$ in Al-doped graphene, the corresponding results are also listed in Table 7. In light of Table 7, the most favorable position with $E_{\mathrm{b}}=-0.427 \mathrm{eV}$ for $\mathrm{H}_{2}$ molecule is shown in Fig. (14b). The distance between $\mathrm{H}_{2}$ and the doped $\mathrm{Al}, l=2.083 \AA$ while that between $\mathrm{H}_{2}$ and carbon layer, $d=2.073 \AA$. As seen from Table 7, the interaction reaches the strongest when both $l$ and $d$ are minimized. The adsorption of $\mathrm{H}_{2}$ in Al-doped graphene is much larger than that in other systems, such as $E_{\mathrm{b}}=-0.41 \mathrm{eV} / \mathrm{H}_{2}$ in Ti- $\mathrm{C}_{2} \mathrm{H}_{4}$-graphene system [107], and $E_{\mathrm{b}}=-0.08 \mathrm{eV} / \mathrm{H}_{2}$ in 12-Li-doped fullerene [108]. However, it still belongs to the physisorption system as the long distance between the doped graphene and the adsorbed $\mathrm{H}_{2}$. Therefore, this strong physisorption interaction would be ideal for hydrogen storage, which adsorbs more $\mathrm{H}_{2}$ molecules.

To understand the enhanced effect of the doped $\mathrm{Al}$ on $\mathrm{H}_{2}$ adsorption, DOSs of the adsorbed $\mathrm{H}_{2}$, the doped $\mathrm{Al}$ and the $\mathrm{C}$ atoms in both $\mathrm{H}_{2}$ /graphene and $\mathrm{H}_{2} / \mathrm{Al}$-doped-graphene systems are plotted and shown in Fig. (15). Fig. (15a) shows DOSs of $\mathrm{H}_{2}$ /graphene system. The main peaks of $\mathrm{H}_{2}$ are located at $-4.37 \mathrm{eV}$ and $6.92 \mathrm{eV}$. However, the main peaks of intrinsic graphene are located between 9 and $13 \mathrm{eV}$. Therefore, the interaction between $\mathrm{H}_{2}$ molecule and the intrinsic graphene is very weak because of non-overlapping of elec-
Table 8. Charges of Atoms in $\mathrm{H}_{2}$ Adsorbed in Graphene System as well as Charge Transfer $Q$ Between Graphene and $\mathrm{H}_{2}$ Molecule, Obtained by Mulliken Analyse. The Unit of the Atom Charge is one Electron Charge $e$, which is Elided Here for Clarity

\begin{tabular}{|c|c|c|}
\hline Atom & Intrinsic Graphene & Al-doped Graphene \\
\hline \hline Al1(C1) & 0.001 & 0.292 \\
\hline C2 & -0.002 & -0.228 \\
\hline C3 & 0 & -0.193 \\
\hline C4 & 0 & -0.193 \\
\hline H5 & -0.001 & -0.001 \\
\hline H6 & -0.001 & 0.021 \\
\hline$Q$ & -0.002 & 0.019 \\
\hline
\end{tabular}

trons in these substances, where $E_{\mathrm{b}}$ is small. On the other hand, for the $\mathrm{H}_{2} / \mathrm{Al}$-doped graphene system shown in Fig. (15b), the main peaks of $\mathrm{H}_{2}$ are located at $-8.15 \mathrm{eV}, 5.74 \mathrm{eV}$, $6.52 \mathrm{eV}$, and $7.51 \mathrm{eV}$, respectively. The bands of $\mathrm{H}_{2}$ interact with both the doped $\mathrm{Al}$ and the $\mathrm{C}$ atoms synchronously at the positions indicated by the dash lines, showing a strong interaction between $\mathrm{H}_{2}$ and Al-doped graphene where $E_{\mathrm{b}}$ is the largest. In addition, the doped $\mathrm{Al}$ changes the electronic structures of both $\mathrm{H}_{2}$ and graphene, and both their DOSs shift towards the lower energy. It exhibits that the $\mathrm{H}_{2} / \mathrm{Al}$-doped-graphene configuration is a much more stable system.

Table $\mathbf{8}$ shows the charge distribution in both $\mathrm{H}_{2}$ /graphene and $\mathrm{H}_{2} / \mathrm{Al}$-doped graphene systems using Mulliken analysis. Before and after $\mathrm{H}_{2}$ adsorption, the charge variation for the former is little while it is significant for the latter. In addition, $\mathrm{H} 6$ has much more positive charge than $\mathrm{H} 5$. Thus, the interaction between $\mathrm{H}_{2}$ and Al-doped graphene is mainly achieved through H6. The interaction between the band at the location of the highest peak of DOS plot of $\mathrm{H}_{2}$ and that of $\mathrm{C}$ atoms implies a strong interaction between the $\mathrm{H}_{2}$ and $\mathrm{C}$ atoms, as shown in Fig. (15b).

The illustrations of electron density distributions for $\mathrm{H}_{2}$ /graphene and $\mathrm{H}_{2}$ /Al-doped graphene systems are shown in Fig. (16). In the former (Fig. (16a)), no electron exists in the region between $\mathrm{H}_{2}$ and $\mathrm{C}$ layer while some electrons appear in the region among $\mathrm{H}_{2}, \mathrm{Al}$ atom and $\mathrm{C}$ layer in the latter (Fig. (16b)). This supports the notion that the $\mathrm{H}_{2}$ /Al-doped graphene possesses a much stronger $\mathrm{H}_{2}$ adsorption ability.

After understanding the enhancement mechanism of $\mathrm{H}_{2}$ adsorption in Al-doped graphene, it is important to determine how many $\mathrm{H}_{2}$ molecules can be adsorbed on the $3 \times 3$ layer surface. We constructed an adsorption configuration with $3 \mathrm{H}_{2}$ molecules adsorbed in the three favorable $\mathrm{C} 1 \mathrm{ad}-$ sorption positions on the topside of the doped system. After geometry relaxation, the atomic structure is shown in Fig. (17a). It has $E_{\mathrm{b}}=-0.303 \mathrm{eV} / \mathrm{H}_{2}$, which is much larger than other systems [104-108] and is within the range of $E_{\mathrm{b}}=-0.20$ $\sim-0.70 \mathrm{eV} / \mathrm{H}_{2}$ at room temperature [102-105] set by the 


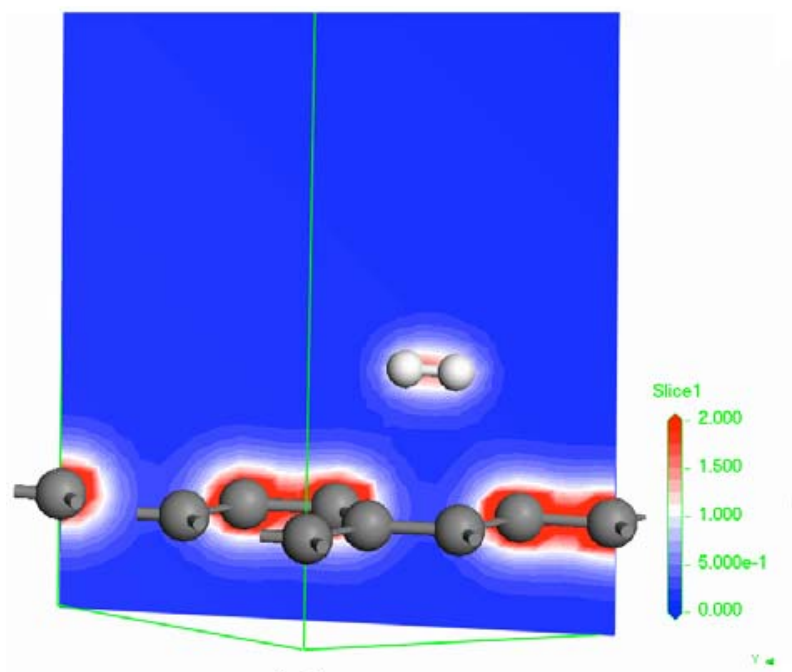

(a)

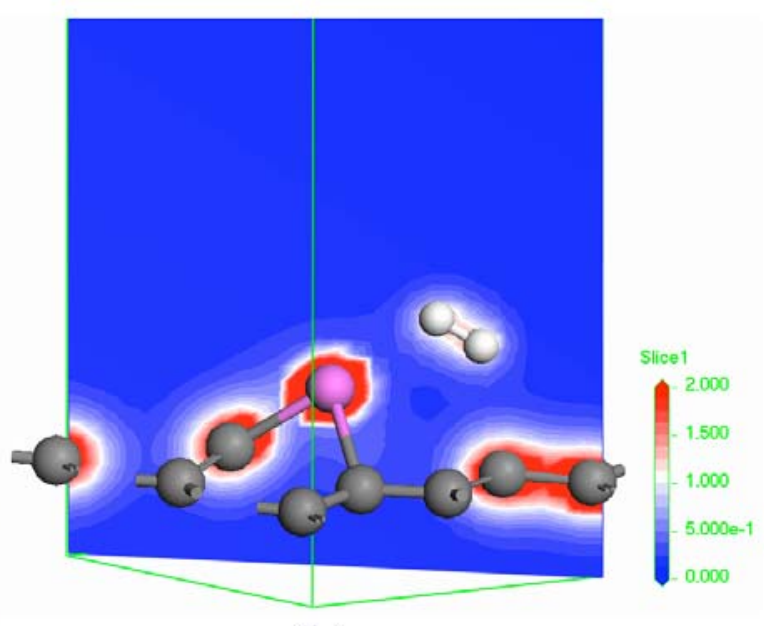

(b)

Fig. (16). Electron density distributions in the $\mathrm{H}_{2} /$ graphene [panel (a)] and $\mathrm{H}_{2} / \mathrm{Al}$-doped-graphene [panel (b)] systems. (Reproduced with permission from Ref. [29]. Copyright 2009, AIP).

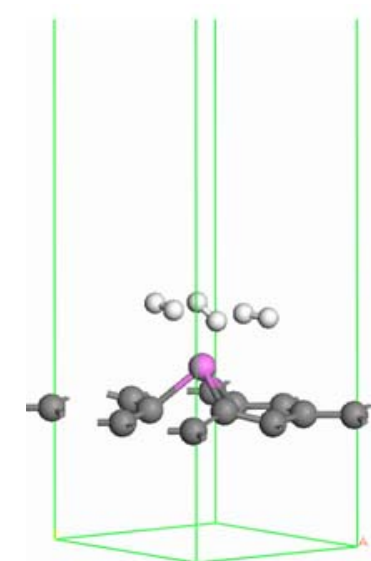

(a)

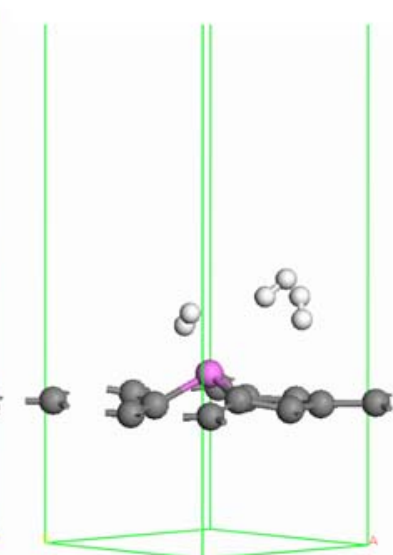

(b)

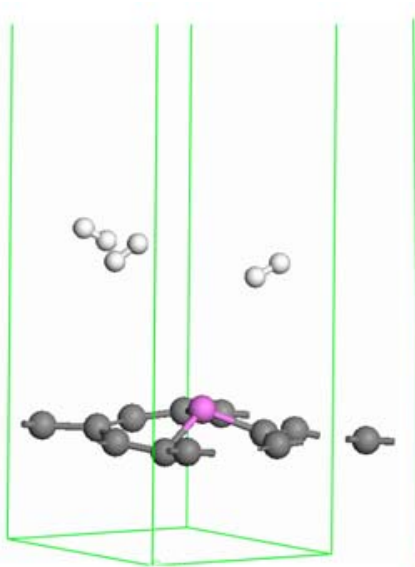

(c)

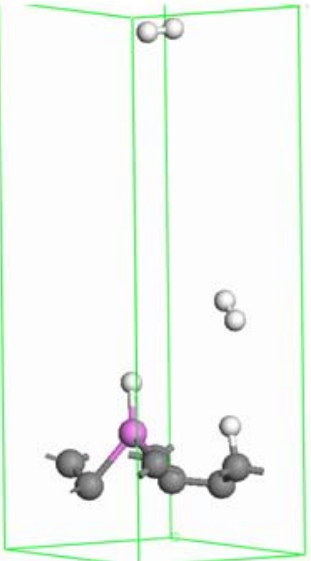

(d)

Fig. (17). Atomic configurations $\mathrm{H}_{2} / \mathrm{Al}$-doped-graphene system at different temperature and pressure. (a) In the ideal condition with $T=0 \mathrm{~K}$, (b) in the condition with $T=300 \mathrm{~K}$ and $P=0.1 \mathrm{GPa}$, (c) in the condition with $T=300 \mathrm{~K}$ and $P=0.0001 \mathrm{GPa}$, and (d) in the condition with $T$ $=300 \mathrm{~K}$ and $P=1 \mathrm{GPa}$. (Reproduced with permission from Ref. [29]. Copyright 2009, AIP).

DOE although the value of $5.1 \mathrm{wt} \%$ of adsorbed $\mathrm{H}_{2}$ is slightly below the DOE's $6 \mathrm{wt} \%$ target.

In order to understand the effect of the number of adsorbed $\mathrm{H}_{2}$ molecules on $E_{\mathrm{b}}$, the configuration with $6 \mathrm{H}_{2}$ molecules adsorbed in Al-doped graphene in the favorable $\mathrm{C} 1$ adsorption sites on both sides was calculated. It is found that $E_{\mathrm{b}}=-0.164 \mathrm{eV} / \mathrm{H}_{2}$, which is almost half of the $E_{\mathrm{b}}$ for the above case where Al-doped graphene adsorbed $3 \mathrm{H}_{2}$ on one side of graphene. In addition, the adsorption with $8 \mathrm{H}_{2}$ molecules in Al-doped graphene was also calculated, and it is found $2 \mathrm{H}_{2}$ molecules were released. In other words, the interaction between $\mathrm{H}_{2}$ molecules would weaken the adsorption on Al-doped graphene and the saturated number of $\mathrm{H}_{2}$ molecules adsorption is 6 . Note that $E_{\mathrm{b}}$ for the cases of $3 \mathrm{H}_{2}$ and $6 \mathrm{H}_{2}$ are respectively $-0.303 \mathrm{eV} / \mathrm{H}_{2}$ and $-0.164 \mathrm{eV} / \mathrm{H}_{2}$, the former is about twice of the latter. This is because $\mathrm{H}_{2}$ molecules were very weakly adsorbed below graphene layer where the doped Al atom locates above graphene layer.

It is well known that $T$ and $P$ have essential effects on hydrogen storage, where increasing $P$ and decreasing $T$ enhance the capacity of hydrogen storage. Thus, the most studied systems are either under high $P$ or at very low $T$ [114], which may not be viable for mobile applications. For example, a storage capacity of $8 \mathrm{wt} \%$ for purified single wall carbon nanotubes (SWNTs) at $80 \mathrm{~K}$ with a hydrogen pressure of $13 \mathrm{MPa}$ [124] and a lower hydrogen storage capacity of 2.3 wt $\%$ at $77 \mathrm{~K}$ were reported [125]. The hydrogen storage capacities in other carbon related materials, such as activated carbon (AC), single walled carbon nanohorn, SWNTs, and graphite nanofibers (GNFs) were also investigated [126]. Although the AC had a capacity of $5.7 \mathrm{wt} \%$ at $77 \mathrm{~K}$ with $P=$ $3 \mathrm{MPa}$, its capacity is < wt1\% at $300 \mathrm{~K}$ [126]. Recent ex- 
perimental results demonstrated that the intrinsic graphene has hydrogen storage capacity of $1.7 \mathrm{wt} \%$ under $1 \mathrm{~atm}$ at 77 $\mathrm{K}$, and $3 \mathrm{wt} \%$ under $100 \mathrm{~atm}$ at $298 \mathrm{~K}$ [127]. Thus, to meet the DOE target, it is necessary to study the adsorption and desorption behaviors of $\mathrm{H}_{2}$ in Al-doped graphene at $T=300$ $\mathrm{K}$ with different $P$. Therefore, the adsorption behaviors of $3 \mathrm{H}_{2}$ /Al-doped-graphene and $6 \mathrm{H}_{2} / \mathrm{Al}$-doped-graphene systems were calculated under $0.0001,0.01,0.1$ and $1 \mathrm{GPa}$ using $a b$ initio $\mathrm{MD}$ simulation. For both the $3 \mathrm{H}_{2} / \mathrm{Al}$-doped graphene and $6 \mathrm{H}_{2} / \mathrm{Al}$-doped graphene systems, we found that all $\mathrm{H}_{2}$ molecules were released at $0.0001 \mathrm{GPa}$ (Fig. (17c)). However, there was only one $\mathrm{H}_{2}$ molecule adsorbed in both systems at $0.01 \mathrm{GPa}$, while the structure of the doped graphene was completely destroyed with $\mathrm{H}$ and $\mathrm{Al}$ forming covalent bond at $1 \mathrm{GPa}$ (Fig. (17d)). When $P=0.1 \mathrm{GPa}$, there are three $\mathrm{H}_{2}$ left on the top side of the two Al-doped systems (Fig. (17b)). Therefore, Al-doped graphene for hydrogen storage capacity at room temperature and $0.1 \mathrm{GPa}$ is 5.13 wt $\%$ with $E_{\mathrm{b}}=-0.260 \mathrm{eV} / \mathrm{H}_{2}$, satisfying the requirements of actual application. In addition, all adsorbed $\mathrm{H}_{2}$ molecules can be released when $P=0.0001 \mathrm{GPa}$.

\section{SUMMARY AND FURTHER PROSPECTS}

The discovery of graphene and its remarkable electronic properties initiated great research interest in this material. Due to the instability of a freestanding graphene, graphene for device applications is generally located on a substrate, which has significant effect on its electrical properties. On the other hand, graphene is a single atomic layer of graphite with surface only; this can maximize the interaction between the surface dopants and adsorbates. Therefore, the properties of the interface and adsorption are important for its potential applications. The main conclusions are following:

1. DFT calculation is employed to study graphene $/ \alpha-\mathrm{SiO}_{2}(0001)$ interface under different $F$. It is found that atomic structure changes slightly except the evident change of $d_{0}$ under $F \leq 0.02$ au. At once $F$ reaches 0.03 au, $\mathrm{C}-\mathrm{O}$ covalent bond in the interface region is present, which deteriorates the electron transport properties of graphene. These results are supported by Mulliken analysis and images of electron density deformation. Thus, the electronic properties of graphene can be modulated by electric fields.

2. A principle to enhance $\mathrm{CO}$ adsorption is developed theoretically using DFT through doping Al into graphene. The results show that Al-doped graphene has strong chemisorption of $\mathrm{CO}$ molecules by forming Al-CO bond, where $\mathrm{CO}$ onto intrinsic graphene remains weak physisorption. Furthermore, the enhancement of CO sensitivity in Al-doped graphene is determined by a large electrical conductivity change after adsorption, where $\mathrm{CO}$ absorption leads to increase of electrical conductivity via introducing large amount of shallow acceptor states. Therefore, this Al-doped graphene would be an excellent candidate for sensing $\mathrm{CO}$ gas. After that, the thermal stability of interaction between the $\mathrm{CO}$ molecules and Al-doped graphene is studied with $a b$ initio molecular dynamics calculation to reveal the adsorption/desorption behaviors of the system. Based on the results of the calculations, the adsorption-desorption phase diagram was established by the atomic thermodynamics and $T$-dependent desorption time $\tau(T)$ was determined with thermal desorption method. The results show that the optimal desorption temperature is $400 \mathrm{~K}$. Meantime, the effect of $T$ on atomic structures and electrical properties are analyzed, and the results show that the greatest conductivity change before and after adsorption with appropriate $\tau$ is at $T=400 \mathrm{~K}$ where the best sensing performance can be achieved.

3. The adsorption behaviors of $\mathrm{H}_{2}$ in the intrinsic and Al-doped graphene using DFT are introduced. The physisorption of $\mathrm{H}_{2}$ is greatly enhanced by doping $\mathrm{Al}$ into graphene. The doped Al varies the electronic structures of both $\mathrm{C}$ and $\mathrm{H}_{2}$, causing the bands of $\mathrm{H}_{2}$ overlapping with those of $\mathrm{Al}$ and $\mathrm{C}$ simultaneously. It induces an intensive interaction between $\mathrm{H}_{2}$ and Al-doped graphene. This was also demonstrated by the electron density distribution. In order to understand effects of $T$ and $P$ on the $\mathrm{H}_{2}$ adsorption behavior for actual application, ab initio MD calculations for $\mathrm{H}_{2}$ /Al-doped graphene system were processed. It is found that the system has $5.13 \mathrm{wt} \%$ hydrogen storage ability at $T=300 \mathrm{~K}$ with $P=$ $0.1 \mathrm{GPa}$. Therefore, Al-doped graphene would be a promising hydrogen storage material.

Apart from its electronic properties, graphene also displays several unusual attributions. Graphene is a giant aromatic macromolecule that conducts both electricity and heat well in two dimensions. Their mechanical strength of graphene is comparable to that of CNTs, while CNTs can be considered graphene with a twist. The shape, size, and chemical structure of graphene sheets can be further modified by engineering. Nonetheless, research toward the application of graphene-based materials has just begun. Many challenges and opportunities remain. For examples, applications for batteries and supercapacitors, in separation technologies, and as supports for catalysts, and so on, are widely expected in the recent future.

\section{ACKNOWLEDGENTS}

The financial support by National Key Basic Research and Development Program (Grant No. 2004CB619301) is acknowledged.

\begin{tabular}{|c|c|c|}
\hline \multicolumn{3}{|c|}{ ABBREVIATIONS } \\
\hline $\mathrm{AC}$ & $=$ & Activated carbon \\
\hline CAPZ & $=$ & Ceperley-Alder-Perdew-Zunger \\
\hline CNT & $=$ & Carbon nanotube \\
\hline DFT & $=$ & Density functional theory \\
\hline DOE & $=$ & U.S. Department of Energy \\
\hline DOS & $=$ & Density of state \\
\hline$d$ & $=$ & Binding distance \\
\hline$d_{0}$ & $=$ & $\begin{array}{l}\text { The equilibrium distance between } \mathrm{SiO}_{2} \\
\text { substrate and graphene overlayer }\end{array}$ \\
\hline$d_{1}$ & $=$ & $\begin{array}{l}\text { The distance between the first and the } \\
\text { second Si atom layers }\end{array}$ \\
\hline DSPP & $=$ & DFT semicore pseudopotentials \\
\hline$E_{\text {ads }}$ & $=$ & Adsorption energy \\
\hline$E_{\mathrm{b}}$ & $=$ & Bond energy \\
\hline
\end{tabular}


$E_{c}$

$E_{\mathrm{f}}$

$E_{\mathrm{g}}$

EG

$E_{\mathrm{GS}}$

$E_{\text {tot }}$

$E_{\mathrm{XC}}$

F

$F[n]$

$F_{\mathrm{X}}$

$G_{\text {ads }}$

$G_{\mathrm{CO}} \quad=$

GEA =

$G_{\mathrm{g}}$

GGA

GNFs

$\Delta G_{\text {ads }}$

$\hat{H}$

$K_{\mathrm{B}}$

$k_{\mathrm{F}}$

$l_{0}$

$l_{\mathrm{Al}-\mathrm{C}}$

LCAO

$l_{\mathrm{C}-\mathrm{C}}$

$l_{\mathrm{C}-\mathrm{O}}$

LDA

MD

$n$

$n_{\mathrm{GS}}$

NPT

NVT

PW91

PBE

$q$

$Q$

RPBE

$s(r)$

$=$

$=$

$=$

$=$

$=$

$=$ $=\quad$ Cutoff energy

$=\quad$ Fermi energy

$=\quad$ Band gap energy

$=\quad$ Epitaxial graphene

$=\quad$ Ground state energy

$=\quad$ Total energy of the system

$=\quad$ Exchange and correlation energy

$=\quad$ Electric field strength

$=\quad$ A functional of the electronic density

$=$ Factor of exchange

$=$ Gibbs free energies of the adsorbed system

Gibbs free energies of the $\mathrm{CO}$ gas molecule

Gradient expansion approximation

Gibbs free energies of Al-doped graphene

$=\quad$ Generalized gradient approximations

$=\quad$ Graphite nanofibers

$=$ Gibbs free energy of adsorption

$=$ Hamiltonian

$=$ Boltzmann's constant

Fermi wavenumber of the material

$=\quad$ The length of Si-O bond at surface or interface

$=\quad$ Bond length of $\mathrm{Al}-\mathrm{C}$ bond

A linear-Combination of Atomic Orbitals

$=\quad$ Average bond length of $\mathrm{C}-\mathrm{C}$

$=\quad \mathrm{C}-\mathrm{O}$ bond length

Local-density approximation

$=\quad$ Molecular dynamics

$=\quad$ Electronic density

$=\quad$ Electronic density of the ground state

Constant numbers of atoms $N$, pressure $P$ and $T$

Constant volume and constant temperature conditions

$=\quad$ Perdew-Wang

$=$ Perdew, Burke and Enzerhof

$=\quad$ Mass parameter

$=\quad$ Net electron transfer

$=$ Revised Perdew-Burke-Emzerhof

$=\quad \mathrm{A}$ dimensionless density gradient parameter

$\begin{array}{lll}\text { SWCNTs } & = & \text { Single wall carbon nanotubes } \\ t & = & \text { Kohn-Sham orbital kinetic energy den- } \\ \text { sities } & & \text { Temperature } \\ \hat{T}_{T} & = & \text { Kinetic interaction operators } \\ T_{0} & = & \text { Kinetic energy } \\ T_{\mathrm{d}} & = & \text { Desorption temperature } \\ \mathrm{TPSS}_{\hat{V}} & = & \text { Tao, Perdew, Staroverov, and Scuseria } \\ V_{e e} & = & \text { Electron-electron interaction operators } \\ V_{\mathrm{ext}} & = & \text { External potential } \\ \varepsilon_{\mathrm{XC}}[n(r)] & = & \text { Exchange-correlation energy density of } \\ \tau(T) & = & \text { a homogeneous electron gas } \\ v_{\mathrm{C}-\mathrm{O}} & = & \text { Vibrational frequency of C-O bond } \\ v_{0} & = & \text { Attempt frequency } \\ \Phi & = & \text { Classical Coulomb potential }\end{array}$

\section{REFERENCES}

[1] Hass, J.; de Heer, W.A.; Conrad, E.H. The growth and morphology of eptitaxial multilayer graphene. J. Phys.: Condens. Matter, 2008, 20, 323202 .

[2] Novoselov, K.S.; Geim, A.K.; Morozov, S.V.; Jiang, D.; Zhang, Y.; Dubonos, S.V.; Grigorieva, I.V.; Firsov, A.A. Electric field effect in atomically thin carbon films. Science, 2004, 306, 666.

[3] Zhang, Y.; Tan, Y-W.; Stormer, H.L.; Kim, P. Experimental observation of the quantum Hall effect and Berry's phase in graphene. Nature, 2005, 438, 201.

[4] Geim, A.K.; Novoselov, K.S. The rise of graphene. Nat. Mater. 2007, 6, 183.

[5] Bunch, J.S.; Yaish, Y.; Brink, M.; Bolotin, K.; McEuen, P.L. Coulomb oscillations and Hall effect in quasi-2D graphite quantum dots. Nano Lett., 2005, 5, 287.

[6] Ao, Z.M.; Zheng, W.T.; Jiang, Q. The effects of electronic field on the atomic structure of the graphene $/ \alpha-\mathrm{SiO}_{2}$ interface. Nanotechnology, 2008, 19, 275710.

[7] Berger, C.; Song, Z.; Li, X.; Wu, X.; Brown, N.; Naud C.; Mayou, D.; Li, T.; Hass, J.; Marchenkov, A.N.; Conrad, E.H.; First, P.N.; de Heer, W.A. Electronic confinement and coherence in patterned epitaxial graphene. Science, 2006, 312, 1191.

[8] Sidorov, A.N.; Yazdanpanah, M.M.; Jalilian, R.; Ouseph, P.J.; Cohn, R.W.; Sumanasekera, G.U. Electrostatic deposition of graphene. Nanotechnology, 2007, 18, 135301.

[9] Zhou, S.Y.; Gweon, G-H.; Fedorov, A.V.; First, P.N.; de Heer, W.A.; Lee, D-H.; Guinea, F.; Castro Neto, A.H.; Lanzara, A. Substrate-induced bandgap opening in epitaxial graphene. Nat. Mater. 2007, 6, 770

[10] Coraux, J.; N'Diaye, A.T.; Busse, C.; Michely, T. Structure coherency of graphene on $\operatorname{Ir}(111)$. Nano Lett. 2008, 8, 565.

[11] Gilje, S.; Han, S.; Wang, M.; Wang, K.L.; Kaner, R.B. A chemical route to graphene for device applications. Nano Lett. 2007, 7, 3394.

[12] Beenakker, C.W.J. Colloquium: Andreev reflection and Klein tunneling in graphene. Rev. Mod. Phys., 2008, 80, 1337.

[13] Ishigami, M.; Chen, J.H.; Cullen, W.G.; Fuhrer, M.S.; Williams, E.D. Atomic structure of graphene on $\mathrm{SiO}_{2}$. Nano Lett. 2007, 7, 1643.

[14] Novoselov, K.S.; Geim, A.K.; Morozov, S.V.; Jiang, D.; Katsnelson, M.I.; Grigorieva, I.V.; Dubonos, S.V.; Firsov, A.A. Two-dimensional gas of massless Dirac fermions in graphene. $\mathrm{Na}$ ture, 2005, 438, 197.

[15] Echtermeyer, T.J.; Lemme, M.C.; Bolten, J.; Baus, M.; Ramsteiner, M.; Kurz, H. Graphene field-effect devices. Eur. Phys. J. Spec. Top., 2007, 148, 19. 
[16] Zhang, Y.; Small, J.P.; Pontius, W.V.; Kim, P. Fabrication and electric-field-dependent transport measurements of mesoscopic graphite devices. Appl. Phys. Lett. 2005, 86, 073104.

[17] Novoselov, K.S.; Jiang, D.; Schedin, F.; Booth, T.J.; Khotkevich, V.V.; Morozov, S.V.; Geim, A.K. Two-dimensional atomic crystals. Proc. Natl. Acad. Sci. USA, 2005, 102, 10451.

[18] Berger, C.; Song, Z.; Li, X.; Ogbazghi, A.Y.; Feng R.; Dai, Z.; Marchenkov, A.N.; Conrad, E.H.; First P.N.; de Heer, W.A. Ultrathin epitaxial graphite: $2 \mathrm{D}$ electron gas properties and a route toward graphene-based nanoelectronics. J. Phys. Chem. B, 2004, 108, 19912.

[19] Wu, X.; Li, X.; Song, Z.; Berger, C.; de Heer, W.A. Weak antiocalization in epitaxial graphene: evidence for chiral electrons. Phys. Rev. Lett., 2007, 98, 136801.

[20] Ando, T.; Nakanishi, T.; Saito, R. Berry's phase and absence of back scattering in carbon nanotubes. J. Phys. Soc. Jpn., 1998, 67, 2857.

[21] Li, D.; Müller, M.B.; Gilje, S.; Kaner, R.B.; Wallace, G.G. Processable aqueous dispersions of graphene nanosheets. Nat. Nanotechnol., 2008, 3, 101.

[22] Chen, H.; Müller, M.B.; Gilmore, K.J.; Wallace, G.G.; Li, D. Mechanically strong, electrically conductive and biocompatible graphene paper. Adv. Mater., 2008, 20, 3557.

[23] Meyer, J.C.; Geim, A.K.; Katsnelson, M.I.; Novoselov, K.S.; Booth, T.J.; Roth, S. The structure of suspended grahene sheets. Nature, 2007, 446, 60 .

[24] Jayaraman, R.; Sodini, C.G. A 1/f noise technique to extract the oxide trap density near the conduction band edge of silicon. IEEE Trans. Electron Devises, 1989, 36, 1773.

[25] Morozov, S.V.; Novoselov, K.S.; Katsnelson, M.I.; Schedin, F.L.; Ponomarenko, A.; Jiang, D.; Geim, A.K. Strong suppression of weak localization in graphene. Phys. Rev. Lett., 2006, 97, 016801.

[26] Giovannetti, G.; Khomyakov, P.A.; Brocks, G.; Kelly, P.J.; van den Brink, J. Substrate-induced band gap in graphene on hexagonal boron nitride: Ab initio density functional calculations. Phys. Rev. B, 2007, 76, 073103.

[27] Okamoto, Y. Density-functional calculations of icosahedral $\mathrm{M}_{13}(\mathrm{M}$ $=\mathrm{Pt}$ and $\mathrm{Au}$ ) clusters on graphene sheets and flakes. Chem. Phys. Lett., 2006, 420, 382.

[28] Ao, Z.M.; Yang, J.; Li, S.; Jiang, Q. Enhancement of CO detection in Al doped graphene. Chem. Phys. Lett., 2008, 461, 276.

[29] Ao, Z.M.; Jiang, Q.; Zhang, R.Q.; Tan, T.T.; Li, S. A doped graphene: A promising material for hydrogen storage at room temperature. J. Appl. Phys. 2009, 105, 074307.

[30] Hwang, E.H.; Adam, S.; Das Sarma, S. Transport in chemically doped graphene in the presence of adsorbed molecules. Phys. Rev. $B, \mathbf{2 0 0 7}, 76,195421$.

[31] Nakada, K.; Fujita, M.; Dresselhaus, G.; Dresselhaus, M.S. Edge state in graphene ribbons: Nanometer size effect and edge shape dependence. Phys. Rev. B, 1996, 54, 17954.

[32] Niimi, Y.; Matsui, T.; Kambara, H.; Tagami, K.; Tsukada, M.; Fukuyama, H. Scanning tunneling microscopy and spectroscopy of the electronic local density of states of graphite surfaces near monoatomic step edges. Phys. Rev. B, 2006, 73, 085421.

[33] Wehling, T.O.; Balatsky, A.V.; Katsnelson, M.I.; Lichtenstein, A.I.; Scharnberg, K.; Wiesendanger, R. Local electronic signatures of impurity states in graphene. Phys. Rev. B, 2007, 75, 125425.

[34] Bertoni, G.; Calmels, L.; Altibeli, A.; Serin, V. First-principles calculation of the electronic structure and EELS spectra at the graphene/Ni(111) interface. Phys. Rev. B, 2005, 71, 075402.

[35] Okamoto, Y. Density-functional calculations of graphene interfaces with $\operatorname{Pt}(111)$ and $\mathrm{Pt}(111) / \mathrm{Ru}_{\mathrm{ML}}$ surfaces. Chem. Phys. Lett., 2005, $407,354$.

[36] N'Diaye, A.T.; Bleikamp, S.; Feibelman, P.J.; Michely, T. Two-dimensional Ir cluster lattice on a graphene Moire on Ir (111). Phys. Rev. Lett., 2006, 97, 215501.

[37] Ao, Z.M.; Li, S.; Jiang, Q. Thermal stability of interaction between the $\mathrm{CO}$ molecules and the Al doped graphene. Phys. Chem. Chem. Phys., 2009, 11, 1683.

[38] Dresselhaus, M.S.; Dresselhaus, G. Intercalation compounds of graphite. Adv. Phys., 2002, 51, 1.

[39] Dutta, P.; Horn, P.M. Low-frequency fluctuations in solids: 1/f noise. Rev. Mod. Phys., 1981, 53, 497.

[40] Lee, C.; Wei, X.; Kysar, J.W.; Hone, J. Measurement of the elastic properties and intrinsic strength of monolayer graphene. Science, 2008, 321, 385 .
[41] Kohn, W.; Sham, L.J. Self-consistent equations including exchange and correlation effects. Phys. Rev., 1965, 140, A1133.

[42] Dirac, P.A.M. Note on exchange phenomena in the Thomas atom. Proc. Cambridge Philos. Soc., 1930, 26, 376.

[43] Perdew, J.P.; Ruzsinszky, A.; Tao, J.; Staroverov, V.N.; Scuseria, G.E.; Csonka, G.I. Prescription for the design and selection of density functional approximations: More constraint satisfaction with fewer fits. J. Chem. Phys., 2005, 123, 062201

[44] Perdew, J.P.; Wang, Y. Accurate and simply density functional for the electronic exchange energy: generalized gradient approximation. Phys. Rev. B, 1986, 33, 8800 .

[45] Becke, A.D. Density-functional exchange-energy approximation with correct asymptotic behavior. Phys. Rev. A, 1988, 38, 3098

[46] Lee, C.; Yang, W.; Parr, G. Development of the Cole-Salvetti correlation-energy formula into a functional of the electron density. Phys. Rev. B, 1988, 37, 785.

[47] Perdew, J.P.; Chevary, J.A.; Vosko, S.H.; Jackson, K.A.; Pederson, M.R.; Singh, D.J.; Fiolhais, C. Atoms, molecules, solids, and surfaces: Applications of the generalized gradient approximation for exchange and correlation. Phys. Rev. B, 1992, 46, 6671.

[48] Perdew, J.P.; Burke, K.; Enzerhof, M. Generalized gradient approximation made simple. Phys. Rev. Lett., 1966, 77, 3865.

[49] Hermann, F.; Van Dyke, J.P.; Ortenburger, I.P. Improved statistical exchange approximation for inhomogeneous many-electron systems. Phys. Rev. Lett., 1969, 22, 807.

[50] Martin, R.M. Electronic Structure: Basic Theory and Practical Methods, Cambridge University Press, UK 2004.

[51] Tao, J.; Perdew, J.P.; Staroverov, V.N.; Scuseria, G.E. Climbing the density functional ladder: Nonempirical meta-generalized gradient approximation designed for molecules and solids. Phys. Rev. Lett., 2003, 91, 146401.

[52] Becke, A.D. Density-functional thermochemistry. III. The role of exact exchange. J. Chem. Phys., 1993, 98, 5648.

[53] Becke, A.D. Density-functional thermochemistry. V. Systematic optimization of exchange-correlation functionals. J. Chem. Phys., 1997, 107, 8554

[54] Moore, G.E. Creamming more components onto integrated circuits. Electronics, 1965, 38, 114

[55] Lin, Y.M.; Appenzeller, J.; Chen, Z.H.; Chen, Z.G.; Cheng, H.M.; Avouris, P. High-performance dual-gate carbon nanotube FETs with 40-nm gate length. IEEE Electron Devices Lett., 2005, 26 , 823 .

[56] Chau, R.; Datta, S.; Doczy, M.; Doyle, B.; Jin, B.; Kavalieros, J.; Majumdar, A.; Metz, M.; Radosavljevic, M. Benchmarking nanotechnology for high-performance and low-power logic transistor applications. IEEE Trans. Nanotechnol., 2005, 4, 153.

[57] Singh, J. Physics of Semiconductors and Their Heterostructures, McGraw-Hill, New York, 1993.

[58] O'Keeffe, J.; Wei, C.Y.; Cho, K.J. Bandstructure modulation for carbon nanotubes in a uniform electric field. Appl. Phys. Lett., 2002, 80,676 .

[59] Li, Y.; Rotkin, S.V.; Ravaioli, U. Electronic response and bandstructure modulation of carbon nanotubes in a transverse electrical field. Nano Lett. 2003, 3, 183.

[60] Fistul, M.V.; Efetov, K.B. Electromagnetic-field-induced suppression of transport through $n-p$ junctions in graphene. Phys. Rev. Lett., 2007, 98, 256803.

[61] Lu, C.L.; Chang, C.P.; Huang, Y.C.; Chen, R.B.; Lin, M.L. Influence of an electric field on the optical properties of few-layer graphene with $A B$ stacking. Phys. Rev. B, 2006, 73, 144427.

[62] Delley, B. From molecules to solids with the DMol ${ }^{3}$ approach. $J$. Chem. Phys., 2000, 113, 7756. Delley, B. Hardness conserving semilocal pseudopotentials. Phys. Rev. B, 2002, 66, 155125.

[63] Hammer, B.; Hansen, L.B.; Nørskov, J.K. Improved adsorption energetics within density-functional theory using revised Perdew-Burke-Ernzerhof functionals. Phys. Rev. B, 1999, 59, 7413.

[64] Liu, W.; Lian, J.S.; Jiang, Q. Theorectical study of $\mathrm{C}_{2} \mathrm{H}_{2}$ adsorbed on low-index Cu surfaces. J. Phys. Chem. C, 2007, 111, 18189.

[65] Aizawa, T.; Souda, R.; Otani, S.; Ishizawa, Y.; Oshima, C. Anomalous bond of monolayer graphite on transition-metal carbide surface. Phys. Rev. Lett., 1990, 64, 768.

[66] Hasegawa, M.; Nishidate, K. Semiempirical approach to the energetics of interlayer binding in graphite. Phys. Rev. B, 2004, 70, 205431.

[67] Peng, S.; Cho, K. Ab initio study of doped carbon nanotube sensors. Nano Lett., 2003, 3, 513. 
[68] Kong, J.; Chapline, M.G.; Dai, H. Functionalized carbon nanotubes for molecular hydrogen sensors. Adv. Mater., 2001, 13, 1384.

[69] Wei, B.Y.; Hsu, M.C.; Su, P.G.; Lin, H.M.; Wu, R.J.; Lai, H.J. A novel $\mathrm{SnO}_{2}$ gas sensor doped with carbon nanotubes operating at temperature. Sensor Actuators B, 2004, 101, 81.

[70] Zhao, Q.; Nardelli, M.B.; Lu, W.; Bernholc, J. Carbon nanotube-metal cluster composites: A new road to chemical sensors?. Nano Lett., 2005, 5, 847.

[71] Wang, R.; Zhang, D.; Sun, W.; Han, Z.; Liu, C. A novel alumimum-doped carbon nanotubes sensor for carbon monoxide. J. Molecular Struct.: Theochem., 2007, 806, 93.

[72] Zhang, Y.M.; Zhang, D.J.; Liu, C.B. Novel chemical sensor for cyanides: Boron-doped carbon nanotubes. J. Phys. Chem. B, 2006, $110,4671$.

[73] Schedin, F.; Geim, A.K.; Morozov, S.V.; Hill, E.W.; Blake, P.; Katasnelson, M.I.; Novoselov, K.S. Detection of individual gas molecules adsorbed on graphene. Nat. Mater., 2007, 6, 652.

[74] Leenaerts, O.; Partoens, B.; Peeters, F.M. Adsorption of $\mathrm{H}_{2} \mathrm{O}, \mathrm{NH}_{3}$, $\mathrm{CO}, \mathrm{NO}_{2}$, and $\mathrm{NO}$ on graphene: A first-principles study. Phys. Rev. $B, \mathbf{2 0 0 8}, 77,125416$.

[75] Wehling, T.O.; Novoselov, K.S.; Morozov, S.V.; Vdovin, E.E.; Katsnelson, M.I.; Geim, A.K.; Lichtenstein, A.I. Molecular doping of graphene. Nano Lett., 2008, 8, 173.

[76] Kong, J.; Franklin, N.R.; Zhou, C.; Chapline, M.G.; Peng, S.; Cho, K.; Dai, H. Nanotube molecular wires as chemical sensors. Science, 2000, 287, 622 .

[77] Collins, P.G.; Bradley, K.; Ishigami, M.; Zettl, A. Extreme oxygen sensitivity of electronic properties of carbon nanotubes. Science, 2000, 287, 1801.

[78] Delley, B. An all-electron numerical method for solving the local density functional for polyatomic molecules. J. Chem. Phys., 1990, 92, 508.

[79] Jeloaica, L.; Sidis, V. DFT investigation of the adsorption of atomic hydrogen on a cluster-model graphite surface. Chem. Phys. Lett., 1999, 300, 157.

[80] Lugo-Solis, A.; Vasiliev, I. Ab initio study of $\mathrm{K}$ adsorption on graphene and carbon nanotubes: Role of long-range ionic forces. Phys. Rev. B, 2007, 76, 235431.

[81] Lide, D.R. CRC Handbook of Chemistry and Physics, 81st ed., CRC Press: Boca Raton, FL, 2000.

[82] Peng, S.; Cho, K.; Qi, P.; Dai, H. Ab initio study of $\mathrm{CNT} \mathrm{NO}_{2}$ gas sensor. Chem. Phys. Lett., 2004, 387, 271.

[83] Hyman, M.P.; Medlin, J.W. Theoretical study of the adsorption and dissociation of oxygen on $\operatorname{Pt}(111)$ in the presence of homogeneous electric fields. J. Phys. Chem. B, 2005, 109, 6304.

[84] Huber, K.P.; Herzberg, G. Molecular Spectra and Molecular Structure, Van Nostrand Reinhold: New York, 1979, vol. IV.

[85] Liao, M.S.; Zhang, Q.E. Electric field-induced shifts of vibrational frequencies of $\mathrm{CO}$ adsorbed on $\mathrm{Ni}, \mathrm{Pd}, \mathrm{Pt}, \mathrm{Cu}, \mathrm{Ag}$ and $\mathrm{Au}$ metal (100) surface: A theoretical comparative study. J. Chem. Soc. Faraday Trans., 1998, 94, 1301.

[86] Verlet, L. Computer "Experiments" on classical fluids. I. Thermodynamical properties of Lennard-Jones molecules. Phys. Rev., 1967, $159,98$.

[87] Suzuki, M. General theory of fractal path integrals with applications to many-body theories and statistical physics. J. Math. Phys., 1991, 32, 400 .

[88] Yoshida, H. Construction of higher order symplectic intergrators. Phys. Lett. A, 1990, 150, 262.

[89] Seitsonen, A.P.; Kim, Y.D.; Knapp, M.; Wendt, S.; Over, H. CO adsorption on the reduced $\mathrm{RuO}_{2}(110)$ surface: Energetics and structure. Phys. Rev. B, 2001, 65, 035413.

[90] Nose, S. A molecular dynamics method for simulations in the canonical ensemble. Mol. Phys., 1984, 52, 255.

[91] Spencer, M.J.S.; Yarovsky, I. Ab initio molecular dynamics study of $\mathrm{H}_{2} \mathrm{~S}$ dissociation on the $\mathrm{Fe}(110)$ surface. J. Phys. Chem. C, 2007, $111,16372$.

[92] Todorova, N.; Spencer, M.J.S.; Yarovsky, I. Ab initio study of S dynamics on iron surfaces. Surf. Sci., 2007, 601, 665.

[93] Loffreda, D. Theoretical insight of adsorption thermodynamics of multifunctional molecules on metal surface. Surf. Sci., 2006, 600, 2103.

[94] Raaen, S.; Ramstad, A. Monte-Carlo simulations of therma desorption of adsorbed molecules from metal surfaces. Energy, 2005, 30, 821.
[95] Readhead, P.A. Thermal desorption of gases. Vacuum, 1962, 12, 203.

[96] Tosatti, E.; Prestipino, S.; Kostlmeier, S.; Dal Corso, A.; Di Tolla, F.D. String tension and stability of magic tip-suspended nanowires. Science, 2001, 291, 288.

[97] Mares, A.I.; Van Ruitenbeek, J.M. Observation of shell effects in nanowires for the nobel metals $\mathrm{Cu}, \mathrm{Ag}$, and Au. Phys. Rev. B, 2005 , 72, 205402.

[98] He, C.; Zhang, P.; Zhu, Y.F.; Jiang, Q. Structures and quantum conduction of copper nanowires under electric field using first principle. J. Phys. Chem. C, 2008, 112, 9045.

[99] Fernández-Sánchez, J.F.; Cannas, R.; Spichiger, S.; Steiger, R.; Spichiger-Keller, U.E. Optical $\mathrm{CO}_{2}$-sensing layers for clinical application based on $\mathrm{pH}$-sensitive indicators incorporated into nanoscopic metal-oxide supports. Sens. Actuators B, 2007, 128, 145.

[100] Coontz, R.; Hanson, B. Not so simple. Science, 2004, 305, 957.

[101] Schlapbach, L.; Züttel, A. Hydrogen-storage materials for mobile applications. Nature, 2001, 414, 353.

[102] Klontzas, E.; Mavrandonakis, A.; Tylianakis, E.; Froudakis, G.E. Improving hydrogen storage capacity of MOF by functionalization of the organic linker with lithium atoms. Nano Lett., 2008, 8, 1572.

[103] Mpourmpakis, G.; Tylianakis, E.; Froudakis, G.E. Carbon nanoscrolls: A promising material for hydrogen storage. Nano Lett., 2007, 7, 1893.

[104] Chandrakumar, K.R.S.; Ghosh, S. Alkali-metal-induced enhancement of hydrogen adsorption in C60 fullerence: An ab initio study. Nano Lett., 2008, 8, 13.

[105] Deng, W.Q.; Xu, X.; Goddard, W.A. New alkali doped pillared carbon materials designed to achieve practical reversible hydrogen storage for transportation. Phys. Rev. Lett., 2004, 92, 166103.

[106] Nikitin, A.; Li, X.; Zhang, Z.; Ogasawara, H.; Dai, H.; Nilsson, A. Hydrogen storage in carbon nanotubes through the formation of stable C-H bonds. Nano Lett., 2008, 8, 162.

[107] Durgun, E.; Ciraci, S.; Zhou, W.; Yildirim, T. Transition-metal-ethylene complexes as high-capacity hydrogen-storage media. Phys. Rev. Lett., 2006, 97, 226102.

[108] Sun, Q.; Jena, P.; Wang, Q.; Marquez, M. First-principles study of hydrogen storage on $\mathrm{Li}_{12} \mathrm{C}_{60}$. J. Am. Chem. Soc., 2006, 128, 9741

[109] Li, X.; Grubisic, A.; Stokes, S.T.; Cordes, J.; Ganteför, G.F.; Bowen, K.H.; Kiran, B.; Willis, M.; Burgert, P.; Schnöckel, H. Unpxpected stability of $\mathrm{Al}_{4} \mathrm{H}_{6}$ : A borane analog?. Science, 2007, 315, 356.

[110] Graetz, J.; Chaudhuri, S.; Lee, Y.; Vogt, T.; Muckerman, J.T.; Reilly, J.J. Pressure-induced structural and electronic changes in $\alpha-\mathrm{AlH}_{3}$. Phys. Rev. B, 2006, 74, 214114.

[111] Cabria, I.; LóPez, M.J.; Alonso, J.A. Hydrogen storage in pure and Li-doped carbon nanopores: Combined effects of concavity and doping. J. Chem. Phys., 2008, 128, 144704.

[112] Okamoto, Y.; Miyamoto, Y. Ab initio investigation of physisorption of molecular hydrogen on planar and curved graphenes. $J$. Phys. Chem. B, 2001, 105, 3470.

[113] Tada, K.; Furuya, S.; Watanabe, K. Ab initio study of hydrogen adsorption to single-walled carbon nanotubes. Phys. Rev. B, 2001, 63,155405 .

[114] Sahaym, U.; Norton, M.G. Advances in the application of nanotechnology in enabling a 'hydrogen economy'. J. Mater. Sci., 2008, 43, 5395.

[115] Girifacol, L.A.; Hodak, M. Van der Waals binding energies in graphite structures. Phys. Rev. B, 2002, 65, 125404.

[116] Krasnov, P.O.; Ding, F.; Singh, A.K.; Yakobson, B.I. Clustering of Sc on SWNT and reduction of hydrogen uptake: Ab-initio all electron calculations. J. Phys. Chem. C, 2007, 111, 179777.

[117] Arellano, J.S.; Molina, L.M.; Rubio, A.; Alonso, J.A. Density functional study of adsorption of molecular hydrogen on graphene layers. J. Chem. Phys., 2000, 112, 8114.

[118] Degall, M.D.; Lindan, P.L.D.; Probert, M.J.; Pickard, C.J.; Hasnip, P.J.; Clark, S.J.; Payne, M.C. First-principles simulation: ideas, illustration and the CASTEP code. J. Phys.: Condens. Matter, 2002, 14, 2717.

[119] Ceperley, D.M.; Alder, B.J. Ground state of the electron gas by a stochastic method. Phys. Rev. Lett., 1980, 45, 566.

[120] Perdew, J.P.; Zunger, A. Self-interaction correction to density-functional approximations for many-electron systems. Phys. Rev. B, 1981, 23, 5048 .

[121] Nose, S. Constant temperature molecular dynamics methods. Prog. Theor. Phys. Suppl., 1991, 103, 1. 
[122] Parrinello, M.; Rahaman, A. Polymorphic transitions in single crystals: A new molecular dynamics method. J. Appl. Phys., 1981, 52,7182 .

[123] Gross, A.; Scheffler, M. Steering and ro-vibrational effects on dissociative adsorption and associative desorption of $\mathrm{H}_{2} / \mathrm{Pd}(100)$. Prog. Surf. Sci., 1996, 53, 187.

[124] Ye,Y.; Ahn, C.C.; Witham, C.; Fultz, B.; Liu, J.; Rinzler, A.G.; Colbert, D.; Smith, K.A.; Smalley, R.E. Hydrogen adsorption and cohesive energy of single-walled carbon nanotubes. Appl. Phys. Lett., 1999, 74, 2307.
[125] Panella, B.; Hirscher, M.; Roth, S. Hydrogen adsorption in different carbon nanostructures. Carbon, 2005, 43, 2209.

[126] Xu, W.C.; Takahashi, K.; Matsuo, Y.; Hattori, Y.; Kumagai, M.; Ishiyama, S.; Kaneko, K.; Iijima, S. Investigation of hydrogen storage capacity of various carbon materials. Int. J. Hydrogen Energy, 2007, 32, 2504.

[127] Ghosh, A.; Subrahmanyam, K.S.; Krishna, K.S.; Datta, S.; Govindaraj, A.; Pati, S.K.; Rao, C.N.R. Uptake of $\mathrm{H}_{2}$ and $\mathrm{CO}_{2}$ by graphene. J. Phys.Chem. C, 2008, 112, 15704.

() Ao and Jiang; Licensee Bentham Open.

This is an open access article licensed under the terms of the Creative Commons Attribution Non-Commercial License (http://creativecommons.org/ licenses/by-nc/3.0/) which permits unrestricted, non-commercial use, distribution and reproduction in any medium, provided the work is properly cited. 